\title{
Local Polynomial Regression with Correlated Errors in Random Design and Unknown Correlation Structure
}

\author{
By K. DE BRABANTER AND F. CAO \\ Department of Statistics, Iowa State University, Ames, Iowa 50011, U.S.A. \\ kbrabant@iastate.edu fancao@iastate.edu \\ AND I. GIJBELS \\ Department of Mathematics, Katholieke Universiteit Leuven, Celestijnenlaan 200B, B-3001 \\ Leuven, Belgium \\ irene.gijbels@wis.kuleuven.be \\ AND J. OPSOMER \\ Department of Statistics, Colorado State University, Fort Collins, Colorado 80523, U.S.A. \\ jopsomer@stat.colostate.edu \\ SUMMARY \\ Automated or data-driven bandwidth selection methods tend to break down in the presence \\ of correlated errors. While this problem has been previously studied in fixed design for kernel \\ regression, the results have only been applicable when there is knowledge on the correlation \\ structure. This article generalizes these results to the random design setting and addresses the \\ problem when no prior knowledge about the correlation structure is available. We establish the \\ asymptotic optimality of our proposed bandwidth selection criterion based on kernels $K$ satisfy- \\ ing $K(0)=0$.
}

Some key words: Autocorrelation; Correlated errors; Cross-validation; Local polynomial regression.

\section{INTRODUCTION}

Assume we observe random variables $\left(X_{i}, Y_{i}\right)_{i=1}^{n}$, where $Y_{i}$ takes values in $\mathbb{R}$ and $X_{i}$ takes values in $\Omega \subseteq \mathbb{R}$ with a common density $f$. The density $f$ is compactly supported, bounded, continuous and $f(x)>0$ for all $x$. Consider the model

$$
Y_{i}=m\left(X_{i}\right)+e_{i}, \quad i=1, \ldots, n,
$$

where $m$ is an unknown smooth regression function and the $e_{i}$ are unobserved random variables with

$$
E\left(e_{i} \mid X_{i}\right)=0, \quad \operatorname{cov}\left(e_{i}, e_{j} \mid X_{i}, X_{j}\right)=\sigma^{2} \rho_{n}\left(X_{i}-X_{j}\right), \quad i, j=1, \ldots, n,
$$

$\sigma^{2}<\infty$ and $\rho_{n}$ is a stationary correlation function satisfying $\rho_{n}(0)=1, \rho_{n}(x)=\rho_{n}(-x)$ and $\left|\rho_{n}(x)\right| \leq 1$ for all $x$. The subscript $n$ allows the correlation function $\rho_{n}$ to shrink as $n \rightarrow \infty$.

Many kernel-based regression methods require the tuning or selection of a smoothing parameter, often referred to as the bandwidth. This introduces arbitrariness into the estimation procedure, so many bandwidth selection methods have been developed (Fan \& Gijbels, 1996; 
Konishi \& Kitagawa, 2008). Diggle \& Hutchinson (1989) and Hart (1991) have shown that these methods perform rather poorly under (1), since they typically require that the errors are independent and identically distributed. Several modifications have been proposed to account for short-range correlation (Opsomer et al., 2001; Francisco-Fernández et al., 2004). The plug-in method of Francisco-Fernández et al. (2004) assumes that the errors are generated from an autoregressive model of order 1, but this could be hard to verify in practice. However, the authors performed a sensitivity analysis suggesting that their methodology is reasonably robust against correlation model misspecification. Lee et al. (2010) proposed two criteria that approximate the averaged squared error when the errors are correlated and the design is fixed. Although their method does not require a parametric form for the correlation structure, it relies on the estimation of the autocorrelation at different lags via the method proposed by Park et al. (2006). Gijbels et al. (1999) considered exponential smoothing and established the relation with nonparametric regression in fixed designs. They assumed that the errors are realizations of a zero-mean causal autoregressive moving average process. Hall \& Van Keilegom (2003) showed that difference methods can be used for autoregressive parameters in nonparametric regression with time series errors. Although the problem of correlated errors is well understood and many remedies exist, a pressing challenge is the implementation of these results (Opsomer et al., 2001).

Kim et al. (2004) developed a nonparametric method to tackle the related problem of detecting correlated errors. They showed that the first-order sample autocovariance of the residuals from kernel regression provides information about whether or not the errors are correlated. Without prior knowledge of the correlation structure, many bandwidth selection procedures are cumbersome to implement. One of the first steps taken to simplify this was proposed by Park et al. (2006), whose estimator of the error correlation is based neither on ordinary cross-validation nor on modified cross-validation (Chu \& Marron, 1991) or block bootstrap (Hall et al., 1995).

To simplify regression with correlated errors, Kim et al. (2009) showed that bimodal kernels are quite effective even when the errors are severely correlated. However, there remain three shortcomings: prior information about the correlated structure is required, bandwidth selection for fixed equispaced designs. In this article we focus on estimating the regression function, not estimating the correlation structure, by local polynomial regression in random designs. As we will show, under mild conditions neither special cross-validation nor prior information about the correlation structure will be required, as a kernel $K$ satisfying $K(0)=0$ can effectively remove the effects of correlation. Since all results are derived for a random design, a natural extension is to consider spatially-correlated errors, which has more applications.

\section{KERNEL SMOOTHER AND ASSUMPTIONS}

If $m$ is an unknown $p+1$ times continuously differentiable function, then the local polynomial regression estimator of order $p$ at an arbitrary point $x$ can be found by solving the following

70 weighted least squares problem. Minimize $\sum_{i=1}^{n}\left\{Y_{i}-\sum_{j=0}^{p} \beta_{j}\left(X_{i}-x\right)^{j}\right\}^{2} K_{h}\left(X_{i}-x\right)$ with respect to $\beta$, yielding

$$
\widehat{\beta}=\left(X_{x}^{T} \mathrm{~W}_{x} X_{x}\right)^{-1} X_{x}^{T} \mathrm{~W}_{x} Y=S_{n}^{-1} X_{x}^{T} \mathrm{~W}_{x} Y
$$


as the solution vector where $Y=\left(Y_{1}, \ldots, Y_{n}\right)^{T}, \mathrm{~W}_{x}$ is the $n \times n$ diagonal matrix of weights $\operatorname{diag}\left\{K_{h}\left(X_{i}-x\right)\right\}$ with kernel $K$ and bandwidth $h, K_{h}(\cdot)=K(\cdot / h) / h, S_{n}=X_{x}^{T} \mathrm{~W}_{x} X_{x}$ and

$$
X_{x}=\left(\begin{array}{ccc}
1\left(X_{1}-x\right) & \cdots & \left(X_{1}-x\right)^{p} \\
\vdots & \vdots & \\
1\left(X_{n}-x\right) & \cdots & \left(X_{n}-x\right)^{p}
\end{array}\right) .
$$

The local polynomial estimator for the regression function at an arbitrary point $x$ is $\widehat{m}(x)=\varepsilon_{1}^{T} \widehat{\beta}$, where $\varepsilon_{1}=(1,0, \ldots, 0)^{T}$ is a unit vector with a 1 on the first position. A useful representation of the local polynomial estimator at an arbitrary point $x$ is

$$
\widehat{m}(x)=\varepsilon_{1}^{T} S_{n}^{-1} X_{x}^{T} \mathrm{~W}_{x} Y=\sum_{i=1}^{n} W_{i}^{n}(x) Y_{i},
$$

with $W_{i}^{n}(x)=\varepsilon_{1}^{T} S_{n}^{-1}\left\{1, X_{i}-x, \ldots,\left(X_{i}-x\right)^{p}\right\}^{T} K\left\{\left(X_{i}-x\right) / h\right\} / h$. Before stating the main theoretical results of the paper, we first give the assumptions on which they rest:

Assumption 1. $m$ is a $p+1$ times continuously differentiable function;

Assumption 2. $h \rightarrow 0$ and $n h \rightarrow \infty$ as $n \rightarrow \infty$;

Assumption 3. there exists an $f_{\max }$ such that $f(x)<f_{\max }$ for all $x \in \Omega \subseteq \mathbb{R}$;

Assumption 4. $f$ has bounded support, continuous and $f(x)>0$ for all $x \in \operatorname{supp}(f)$;

Assumption 5. there exists a constant $K_{\max }$ such that $|K(x)|<K_{\max }, K(x) \geq 0$ for all $x$;

Assumption 6. $K$ is symmetric and Lipschitz continuous at 0 ;

Assumption 7. $\lim _{|u| \rightarrow \infty}|u|^{l} K(u)<\infty$ with $l=0, \ldots, p$;

Assumption 8. the correlation function $\rho_{n}$ is an element of a sequence $\left\{\rho_{n}\right\}$ with the following properties for all $n$. There exist constants $\rho_{\max }$ and $\rho_{c}$ such that $n \int\left|\rho_{n}(x)\right| d x<\rho_{\max }$ and $\lim _{n \rightarrow \infty} n \int \rho_{n}(x) d x=\rho_{c}$. For any sequence $\epsilon_{n}>0$ satisfying $n \epsilon_{n} \rightarrow \infty$,

$$
n \int_{|x| \geq \epsilon_{n}}\left|\rho_{n}(x)\right| d x \rightarrow 0, \quad n \rightarrow \infty ;
$$

Assumption 9. $\lim _{n \rightarrow \infty} n \int \rho_{n}^{2}(x) d x=\rho_{c c}$; and

Assumption 10. for all $i, j, k, l$,

$$
\begin{aligned}
\operatorname{cov}\left(e_{i} e_{j}, e_{k} e_{l} \mid X_{i}, X_{j}, X_{k}, X_{l}\right)= & \operatorname{cov}\left(e_{i}, e_{k} \mid X_{i}, X_{k}\right) \operatorname{cov}\left(e_{j}, e_{l} \mid X_{j}, X_{l}\right) \\
& +\operatorname{cov}\left(e_{i}, e_{l} \mid X_{i}, X_{l}\right) \operatorname{cov}\left(e_{j}, e_{k} \mid X_{j}, X_{k}\right) .
\end{aligned}
$$

Assumption 4 requires a density $f$ with bounded support. Strictly speaking, this means that distributions defined on the entire space $\mathbb{R}$ are excluded but our simulations suggest that the proposed method also works when the design points are sampled from densities with infinite support. Assumption 8 implies that the correlation function depends on $n$ and that $\int\left|\rho_{n}(x)\right| d x$ should vanish at a rate not slower than $O(1 / n)$. We will also require the correlation to be shortrange, in the second part of Assumption 8. Two correlation functions satisfying Assumption 8 are (Cressie, 1993)

$$
\rho_{n}(x)=\exp (-\alpha n|x|), \quad \rho_{n}(x)=\frac{1}{1+\alpha n^{2} x^{2}}, \quad \alpha>0 .
$$


These common assumptions are generalizations of the equispaced design assumptions in Altman (1990). Assumptions 8 and 10 are satisfied, for example, when the errors have an autoregressive

100

with

$$
\begin{aligned}
\operatorname{var}\left\{\widehat{m}(x) \mid X_{1}, \ldots, X_{n}\right\} & =\frac{\sigma^{2}\left\{1+f(x) \rho_{c}\right\}}{n h f(x)} \varepsilon_{1}^{T} S^{-1} S^{\star} S^{-1} \varepsilon_{1}+o_{p}\left(\frac{1}{n h}\right) \\
& =\int K_{p}^{\star 2}(t) d t \frac{\sigma^{2}\left\{1+f(x) \rho_{c}\right\}}{n h f(x)}+o_{p}\left(\frac{1}{n h}\right),
\end{aligned}
$$

where $S=\left(\mu_{i+j}\right)_{0 \leq i, j \leq p} \quad$ with $\quad \mu_{j}=\int u^{j} K(u) d u, \quad S^{\star}=\left(\nu_{i+j}\right)_{0 \leq i, j \leq p} \quad$ with $\quad \nu_{j}=$ $\int u^{j} K^{2}(u) d u, c_{p}=\left(\mu_{p+1}, \ldots, \mu_{2 p+1}\right)^{T}$ and $K_{p}^{\star}(t)=\varepsilon_{1}^{T} S^{-1}\left(1, t, \ldots, t^{p}\right)^{T} K(t)$.

The bandwidth $h_{\mathrm{opt}}$ minimizing the asymptotic mean integrated squared error, i.e., $\int\left[\operatorname{bias}^{2}\left\{\widehat{m}(x) \mid X_{1}, \ldots, X_{n}\right\}+\operatorname{var}\left\{\widehat{m}(x) \mid X_{1}, \ldots, X_{n}\right\}\right] f(x) d x$ readily follows and is given in Corollary 1 . There is a slight but significant difference between the correlated and independent error cases (Fan \& Gijbels, 1996, p. 68).

COROLLARY 1. Under the assumptions of Theorem 1, the asymptotically optimal constant bandwidth for $p$ odd is

$$
h_{o p t}=C_{p}(K)\left[\frac{\sigma^{2}\left\{\mu(\Omega)+\rho_{c}\right\}}{\int\left\{m^{(p+1)}(x)\right\}^{2} f(x) d x}\right]^{1 /(2 p+3)} n^{-1 /(2 p+3)},
$$

$$
C_{p}(K)=\left[\frac{(p+1) !^{2} \int K_{p}^{\star 2}(t) d t}{2(p+1)\left\{\int t^{p+1} K_{p}^{\star}(t) d t\right\}^{2}}\right]^{1 /(2 p+3)}
$$

and $\mu(\Omega)$ denotes the Lebesgue measure of the design region $\Omega \subseteq \mathbb{R}$.

In what follows define the residual sum of squares $\operatorname{RSS}(h)=n^{-1} \sum_{i=1}^{n}\left\{Y_{i}-\widehat{m}\left(X_{i}\right)\right\}^{2}$ and the asymptotic squared error $\operatorname{ASE}(h)=n^{-1} \sum_{i=1}^{n}\left\{m\left(X_{i}\right)-\widehat{m}\left(X_{i}\right)\right\}^{2}$. Next, we will show that $\operatorname{RSS}(h)$ approximates $\operatorname{ASE}(h)$ well enough uniformly over a set of bandwidths. To prove this 
result, we require a slightly stronger condition on the correlation function than that in Assumption 8 . However, the correlation functions in (2) also satisfy this stronger condition.

THEOREM 2. Under Assumptions 1 to 10 and if $n^{\delta} \int\left|\rho_{n}(t)\right| d t<\rho_{\delta}$ for $\delta>1$, $p$ odd and $h \in \mathcal{H}_{n}$ where $\mathcal{H}_{n}=\left[a n^{-1 /(2 p+3)}, b n^{-1 /(2 p+3)}\right], 0<a<b<\infty$ then

$$
\operatorname{RSS}(h)=\operatorname{ASE}(h)+\frac{1}{n} \sum_{i=1}^{n} e_{i}^{2}-\frac{2 \sigma^{2} K(0) S^{00}\left\{\mu(\Omega)+\rho_{c}\right\}}{n h}+o_{p}\left\{n^{-(2 p+2) /(2 p+3)}\right\},
$$

where $S^{00}$ denotes the first element of the first row of the inverse of the matrix $S$.

Theorem 3 establishes our proposed bandwidth selection criterion based on residual sum of squares for correlated data and unknown correlation structure. Theorem 3 shows that the proposed criterion results in an optimal bandwidth that minimizes the asymptotic squared error asymptotically.

THEOREM 3. Assume the assumptions of Theorem 2 hold. Let $\widehat{h}_{\mathrm{RSS}}$ be the minimizer of the residual sum of squares criterion based on a kernel satisfying $K(0)=0$ over $h \in \mathcal{H}_{n}$. Further, let $\widehat{h}_{\mathrm{ASE}}$ be the minimizer of the asymptotic squared error over $h \in \mathcal{H}_{n}$. Then uniformly for $h \in \mathcal{H}_{n}$

$$
\operatorname{RSS}(h)=\frac{1}{n} \sum_{i=1}^{n} e_{i}^{2}+\operatorname{ASE}(h)+o_{p}\left\{n^{-(2 p+2) /(2 p+3)}\right\}
$$

and $\operatorname{ASE}\left(\widehat{h}_{\mathrm{RSS}}\right) / \operatorname{ASE}\left(\widehat{h}_{\mathrm{ASE}}\right)=1+o_{p}(1)$, or equivalently as $n \rightarrow \infty$

$$
\frac{\operatorname{ASE}\left(\widehat{h}_{\mathrm{RSS}}\right)}{\inf _{h \in \mathcal{H}_{n}} \operatorname{ASE}(h)} \rightarrow 1 \quad \text { in probability. }
$$

Proof. From Theorem 2 and choosing a kernel satisfying $K(0)=0$, it follows that

$$
\operatorname{RSS}(h)=\frac{1}{n} \sum_{i=1}^{n} e_{i}^{2}+\operatorname{ASE}(h)+o_{p}\left\{n^{-(2 p+2) /(2 p+3)}\right\} .
$$

Next,

$$
\begin{aligned}
\operatorname{ASE}\left(\widehat{h}_{\mathrm{RSS}}\right) & =\operatorname{RSS}\left(\widehat{h}_{\mathrm{RSS}}\right)-\frac{1}{n} \sum_{i=1}^{n} e_{i}^{2}+o_{p}\left\{n^{-(2 p+2) /(2 p+3)}\right\} \\
& \leq \operatorname{RSS}\left(\widehat{h}_{\mathrm{ASE}}\right)-\frac{1}{n} \sum_{i=1}^{n} e_{i}^{2}+o_{p}\left\{n^{-(2 p+2) /(2 p+3)}\right\} \\
& =\operatorname{ASE}\left(\widehat{h}_{\mathrm{ASE}}\right)+o_{p}\left\{n^{-(2 p+2) /(2 p+3)}\right\} .
\end{aligned}
$$

By definition of $\widehat{h}_{\mathrm{ASE}}$ it follows that $\operatorname{ASE}\left(\widehat{h}_{\mathrm{ASE}}\right) \leq \mathrm{ASE}\left(\widehat{h}_{\mathrm{RSS}}\right)$. Asymptotic optimality (Li, ${ }_{140}$ 1987) of our bandwidth selection criterion is a consequence of (5) and Theorem 2.

Theorem 3 states that minimizing $\operatorname{ASE}(h)$ is asymptotically equivalent to minimizing $\operatorname{RSS}(h)$ with a kernel satisfying $K(0)=0$. We need not estimate anything regarding the correlation. This type of kernel successfully removes the effects of correlation and eliminates the need for cross-validation-based techniques, since it puts a zero value for the point under consideration, ${ }_{145}$ automatically creating a leave-one-out cross-validation scenario. 

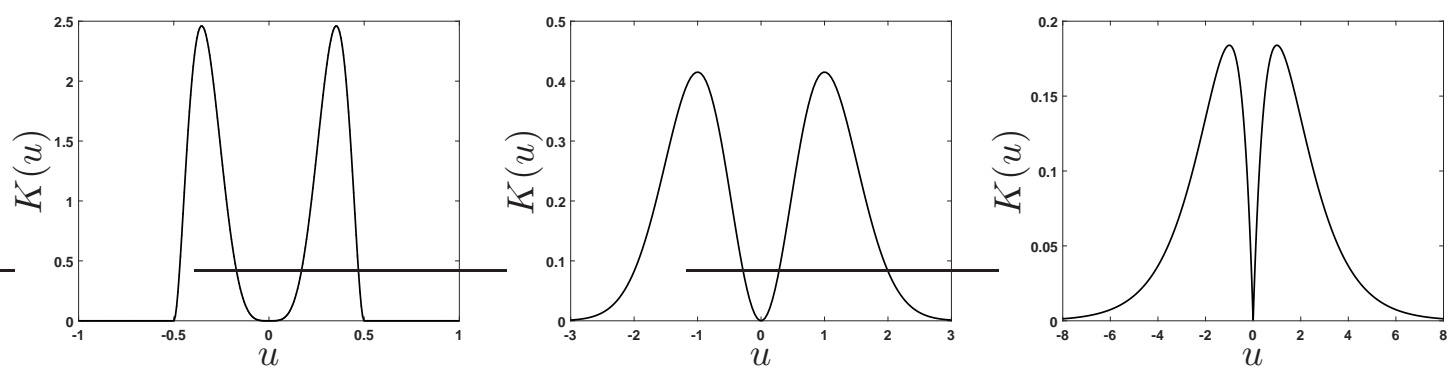

Fig. 1: Three kernels satisfying $K(0)=0$. Note the difference around zero for these kernels. $K(u)=630\left(4 u^{2}-1\right)^{2} u^{4} I_{(|u| \leq 1 / 2)}$ (left), $K(u)=2 \pi^{-1 / 2} u^{2} \exp \left(-u^{2}\right)$ (middle) and $K(u)=$ $\frac{1}{2}|u| \exp (-|u|)$ (right).

\section{KERNEL AND BANDWIDTH SELECTION IN PRACTICE}

Although kernels satisfying $K(0)=0$ are effective in removing the effects of correlation in bandwidth selection, they also introduce extra bias and variability (Kim et al., 2009). An optimal kernel $\tilde{K}$ in this framework, ignoring Assumption 6, would be

$$
\tilde{K}(u)= \begin{cases}\frac{3}{4}\left(1-u^{2}\right), & |u| \leq 1 \\ 0, & u=0\end{cases}
$$

the Epanechnikov kernel with a discontinuity at zero, but the latter condition violates Assumption 6. It is easy to see that an optimal kernel does not exist in the class of kernels satisfying $K(0)=0$ and Assumption 6 (Kim et al., 2009). The Epanechnikov kernel is obtained by minimizing the following quantity with respect to $K$, with extra constraints omitted here,

$$
Q_{K}=\left\{\int u^{2} K(u) d u\right\}\left\{\int K^{2}(u) d u\right\}^{2} .
$$

The value $Q_{K}$ for the Epanechnikov kernel is 0.072. For $K(u)=630\left(4 u^{2}-1\right)^{2} u^{4} I_{(|u| \leq 1 / 2)}$, the kernel used by Kim et al. (2009), $Q_{K}=0 \cdot 374$. As a result, this kernel is roughly five times less efficient than the Epanechnikov. Next, we will introduce two kernels with $Q_{K}$ values closer to the Epanechnikov's. The kernels $K(u)=2 \pi^{-1 / 2} u^{2} \exp \left(-u^{2}\right)$ and $K(u)=2^{-1}|u| \exp (-|u|)$ have $Q_{K}$ values 0.134 and 0.093 respectively. The three kernels discussed here, shown in Figure 1, exhibit very different behavior around zero. All three satisfy Assumption 6. Among these three kernels satisfying $K(0)=0$, we recommend the use of $K(u)=2 \pi^{-1 / 2} u^{2} \exp \left(-u^{2}\right)$, whose smooth trough around zero makes finding the minimum of the residual sum of squares more numerically stable. Although finding an optimal kernel is an interesting question, we do not pursue it here.

Kernels with $K(0)=0$ are very effective in removing the unknown correlation, but due to their non-optimality, they introduce extra mean squared error, producing a more wiggly estimate of the regression function. To counteract this and as our final smoothing procedure, we propose that the kernel with $K(0)=0$ is used to provide a pilot bandwidth $\widehat{h}_{\text {bimd }}$ that can then be related to that of the bandwidth of a unimodal kernel via a so-called factor rule. In order to distinguish between a kernel satisfying $K(0)=0$ and a unimodal kernel, we will denote kernels satisfying $K(0)=0$ by $\bar{K}$. Both $K$ and $\bar{K}$ should satisfy Assumption 6. Corollary 1 suggests that the relation between the bandwidth of a kernel $\bar{K}$ with bandwidth $\widehat{h}_{\text {bimd }}$ and Gaussian kernel $K$ with 
bandwidth $\widehat{h}$ is

$$
\widehat{h}=\frac{C_{p}(K)}{C_{p}(\bar{K})} \widehat{h}_{\text {bimd }}=C_{p}(K, \bar{K}) \widehat{h}_{\text {bimd }}
$$

where

$$
C_{p}(K, \bar{K})=\left[\frac{\int K_{p}^{\star 2}(t) d t\left\{\int t^{p+1} \bar{K}_{p}^{\star}(t) d t\right\}^{2}}{\int \bar{K}_{p}^{\star 2}(t) d t\left\{\int t^{p+1} K_{p}^{\star}(t) d t\right\}^{2}}\right]^{1 /(2 p+3)} .
$$

The equivalent kernel $\bar{K}_{p}^{\star}$ can be easily obtained by substituting $\bar{K}$ for $K$. Values for $C_{p}(K, \bar{K}) \quad{ }_{175}$ are given in the next section.

\section{Simulations}

Below we compare the effect on bandwidth selection of using the Gaussian kernel with that of a kernel satisfying $K(0)=0$. Since the use of such kernels introduces extra variance, we perform a two-step procedure to reduce the variance. First, we use the kernel $\bar{K}(u)=$ $2 \pi^{-1 / 2} u^{2} \exp \left(-u^{2}\right)$ to obtain $\widehat{h}_{\text {bimd }}$, which is in fact $\widehat{h}_{\mathrm{RSS}}$. Second, we exploit the asymptotic relationship between the optimal bandwidths to get to $\widehat{h}$, as indicated in expression (6). This requires no extra smoothing and the relation between the kernel satisfying $K(0)=0$ and the Gaussian kernel $K$ is given by (6) with $C_{p}(K, \bar{K})=1.16$ or $C_{p}(K, \bar{K})=1.01$ for local linear, $p=1$, or local cubic, $p=3$, regression respectively. The Epanechnikov kernel, optimal in $L_{2}$, could have been taken as unimodal kernel in the second step, but since there is not much difference in optimality between the Gaussian $Q_{k}=0.08$ and the Epanechnikov kernel $Q_{k}=0.072$ and due to the link between the shapes of the kernel satisfying $K(0)=0$ and Gaussian kernel, we chose the Gaussian kernel in the second step. In the simulation study we used local linear regression. We also compare with standard cross-validation using the Gaussian kernel ignoring correlation among the errors, and the plug-in method proposed by Francisco-Fernández et al. (2004) which involves estimating the correlation structure of the errors; it was assumed that the design is regular and generated by a density. We use equally-spaced designs in the simulations.

Consider the model $Y_{i}=m\left(x_{i}\right)+e_{i}$ where $x_{i}=i / n, m\left(x_{i}\right)=2 \sin \left(6 x_{i}\right)+\left(x_{i}+1\right)^{2}, e_{i} \sim$ $N(0,1)$ and $\operatorname{cov}\left(e_{i}, e_{j}\right)=\sigma^{2} \rho\left(\left|x_{i}-x_{j}\right|\right)$. Also, consider two correlation functions $\rho_{1}(t)=$ $\exp (-\alpha|t|), \alpha=50,100,200,1000$, for the autoregressive model of order 1 , and

$$
\rho_{2}(t)=\exp \left(-\alpha_{1} t / 2\right) \cos (p t)+\frac{\alpha_{1}}{2 p} \sin (p t),
$$

where $p^{2}=\left(\alpha_{2}-\alpha_{1}^{2} / 4\right)>0$ for a quasi-periodic autoregressive model of order two correlation structure. We use $\left(\alpha_{1}, \alpha_{2}\right)=\{(50,3000),(50,4000),(60,3000),(60,4000)\}$ for $\rho_{n 2}$. The correlation functions are shown in Figure 2 and Figure 3. For each of the correlation structures we generate 5000 sample data replicates and compute the estimated bandwidth $\widehat{h}$ using the three estimation methods mentioned earlier for sample sizes $n=50,100,200$. We approximate the value for the mean of $\widehat{h}_{\mathrm{ASE}}$ for 5000 data replicates denoted by $h_{\mathrm{MASE}}$. In general, the bandwidth density of our method is centered at zero with variance comparable to the plug-in and cross-validation methods.

With positive autoregressive correlated errors of order one, the plug-in method is resistant to correlation and usually performs better than cross-validation with a normal kernel. The bandwidth density of the proposed method is centered at zero, so our method will give better estimates of the optimal bandwidth. When $\alpha=1000$, which is almost the independent error case, all the 

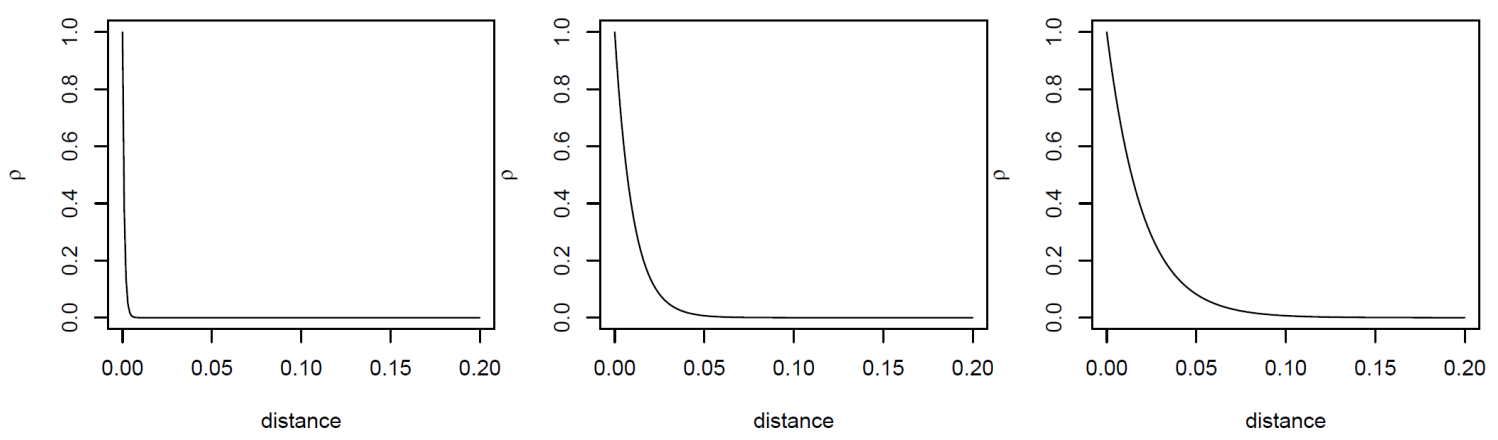

Fig. 2: Correlation function $\rho_{1}(\cdot)$ for three different parameter settings. $\alpha=1000$ (left), $\alpha=100$ (middle) and $\alpha=50$ (right).
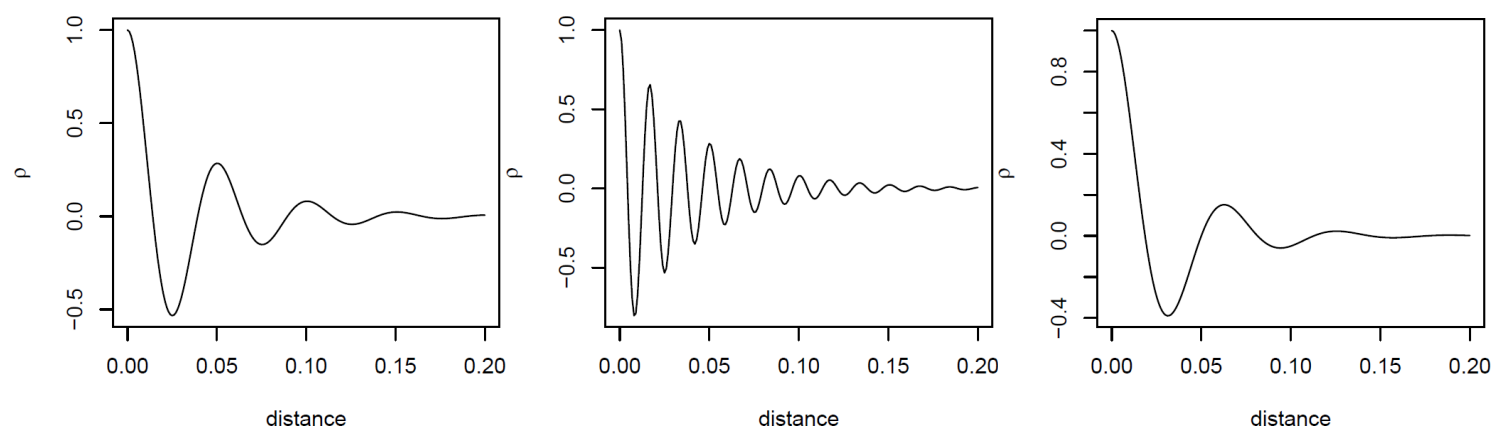

Fig. 3: Correlation function $\rho_{2}(\cdot)$ for three different parameter settings. $\left(\alpha_{1}=50\right.$ and $\left.\alpha_{2}=3000\right)$ (left), $\left(\alpha_{1}=50\right.$ and $\left.\alpha_{2}=4000\right)$ (middle) and $\left(\alpha_{1}=60\right.$ and $\left.\alpha_{2}=4000\right)$ (right).

methods give good results, but our method has a slight variance increase. When $\alpha$ decreases, the correlation increases, and the other two methods tend to give smaller bandwidths than optimal. However, for the plug-in method to work, a pilot smooth is needed. Since Francisco-Fernández et al. (2004) did not specify practical guidelines on how to choose this, we used our method based on a kernel satisfying $K(0)=0$ as pilot smooth to obtain residuals. The results for $n=100$ are shown in Figure 4; see also the Supplementary Material. The results for the autoregressive model, shown in Figure 5, indicate that our method will generally outperform the plug-in method and standard cross-validation. It shows not only the ability to perform well with positive correlation, but also with negative correlation.

\section{DISCUSSION}

Let $\left(X, Y_{i}\right)_{i=1}^{n}$ be a set of $\mathbb{R}^{d+1}$-valued random vectors, where the $Y_{i}$ are scalar responses and 220 the $X_{i}$ are $\mathbb{R}^{d}$-valued covariates with common density $f$ with compact support $\Omega \subseteq \mathbb{R}^{d}$. Further, $f$ is bounded, continuous and $f(x)>0$ for all $x$. Consider the same model as in Section 1 with $\rho_{n}$ a $d$-variate stationary correlation function. The local linear estimator for $m(\cdot)$ at $x$ is the 

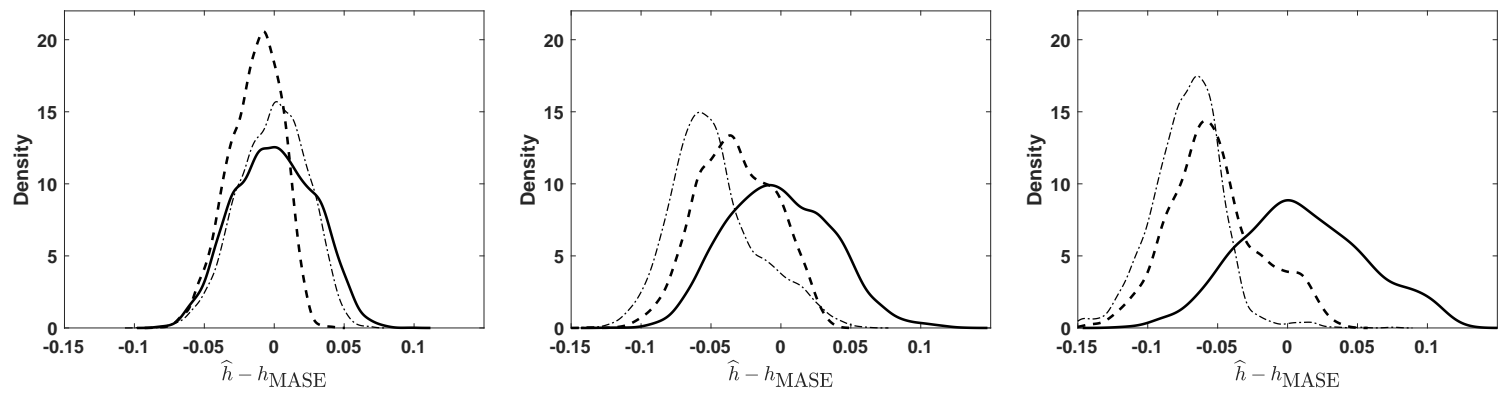

Fig. 4: Densities of the bandwidth estimators $\widehat{h}$ (solid line), plug-in bandwidth $\widehat{h}_{\text {plgn }}$ (dashed line) and cross-validation ignoring correlation $\widehat{h}_{\text {norm }}$ (dash dotted line) for autoregressive model of order one correlated errors and $n=100$ with $\alpha=1000$ (left), $\alpha=100$ (middle) and $\alpha=50$ (right). The result for $\alpha=200$ is omitted.
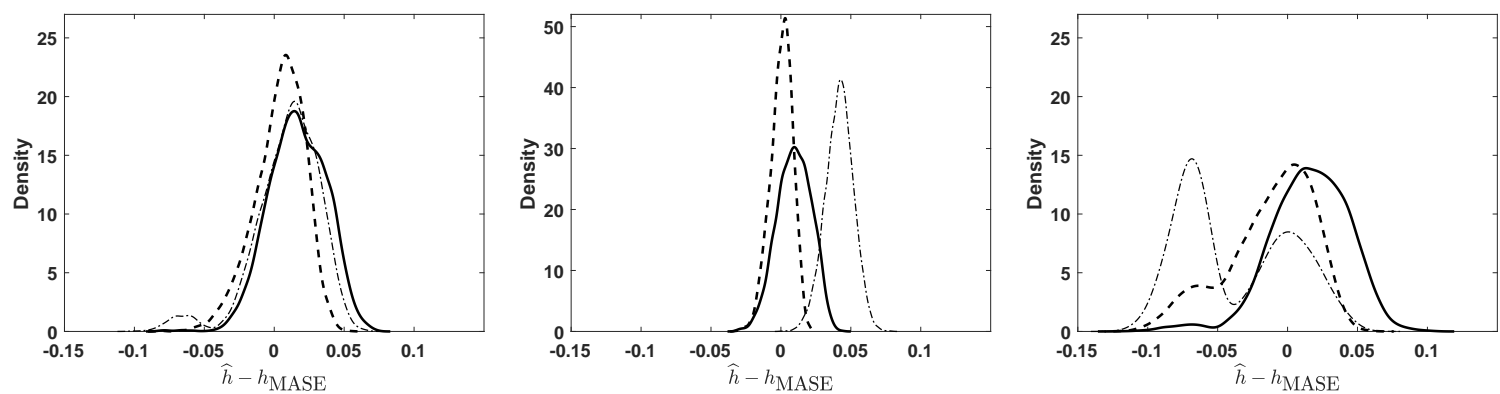

Fig. 5: Densities of the bandwidth estimators $\widehat{h}$ (solid line), plug-in bandwidth $\widehat{h}_{\text {plgn }}$ (dashed line) and cross-validation ignoring correlation $\widehat{h}_{\text {norm }}$ (dash dotted line) for autoregressive model of order two correlated errors and $n=100$ with $\alpha_{1}=50, \alpha_{2}=3000$ (left), $\alpha_{1}=50, \alpha_{2}=4000$ (middle) and $\alpha_{1}=60, \alpha_{2}=4000$ (right). The panel in the middle has a different scale on the vertical axis. The result for $\alpha_{1}=60, \alpha_{2}=3000$ is omitted.

solution to the least squares minimization

$$
\min _{\alpha, \beta} \sum_{i=1}^{n}\left\{Y_{i}-\alpha-\beta^{T}\left(X_{i}-x\right)\right\}^{2} K_{H}\left(X_{i}-x\right)
$$

with $H$ a $d \times d$ symmetric positive definite bandwidth matrix, $K$ a $d$-variate kernel and $K_{H}(u)=$ $|H|^{-1} K\left(H^{-1} u\right)$ with $|H|$ the determinant of the matrix $H$. A key in extending our methodology to the multivariate case is the results of Ruppert \& Wand (1994) for the asymptotic expression of $S_{n}^{-1}=\left(X_{x}^{T} \mathrm{~W}_{x} X_{x}\right)^{-1}$. In what follows we write $\lambda_{\max }(H)$ and $\lambda_{\min }(H)$ for the maximal and minimal eigenvalues of the matrix $H$ and $\|x\|$ for the $L_{2}$-norm. Finally, for a matrix $H$, $H \rightarrow 0$ implies that every element of $H$ goes to zero. Besides the assumptions on the density $f$, regression function $m$ and kernel $K$, we also require that the bandwidth matrix $H$ be symmetric and positive definite. Next, $H \rightarrow 0$ and $n|H| \rightarrow \infty$ as $n \rightarrow \infty$, the ratio $\lambda_{\max }(H) / \lambda_{\min }(H)$ is bounded above. Next, $\lim _{\|u\| \rightarrow \infty}\|u\|^{l} K(u)<\infty$ with $l=0,1$. Finally, the first part of Assumption 8, Assumptions 9 to 10 for $\rho_{n}$ still hold, with the second part of Assumption 8 replaced by: 
for any sequence $\epsilon_{n}>0$ satisfying $n^{1 / d} \epsilon_{n} \rightarrow \infty$

$$
n \int_{\|x\| \geq \epsilon_{n}}\left|\rho_{n}(x)\right| d x \rightarrow 0, \quad n \rightarrow \infty .
$$

235

Then for $n^{\delta} \int\left|\rho_{n}(t)\right| d t<\rho_{\delta}$ and $\delta>1$, each element of $H$ is $O\left(n^{-1 /(d+4)}\right)$ and a kernel $K$ for which $K(0)=0$ Theorem 2 is extended to the multivariate case. A possible kernel could be $K(u)=\left(2 d^{-1} \pi^{-d / 2}\right)\|u\|^{2} \exp \left(-\|u\|^{2}\right)$. Two commonly used correlation functions satisfying Assumption 16 are the exponential and rational quadratic models (Cressie, 1993)

$$
\rho_{n}(x)=\exp \left(-\alpha n^{1 / d}\|x\|\right), \quad \rho_{n}(x)=\frac{1}{1+\alpha n^{2 / d}\|x\|^{2}}, \quad \alpha>0 .
$$

\section{ACKNOWLEDGEMENT}

We are very grateful to the associate editor and referees for their helpful comments. The third author gratefully acknowledges support from the Research Fund Katholieke Universiteit Leuven, and from the Interuniversity Attraction Poles Research Network P7/06 of the Belgian Science Policy.

\section{SUPPLEMENTARY MATERIAL}

Supplementary material available at Biometrika online includes the proofs of Theorems 1 and 2 and additional simulation results.

\section{REFERENCES}

De Brabanter K., De Brabanter J., Suykens J.A.K. \& De Moor B. (2011). Kernel Regression in the Presence of Correlated Errors. J. Mach. Learn. Res., 12, 1955-1976.

250 Altman, N.S. (1990). Kernel smoothing of data with correlated errors. J. Amer. Statist. Assoc. 84, 749-759.

ChU, C.K. \& MARron J.S. (1991). Comparison of two bandwidth selectors with dependent errors. Ann. Statist. 4 , 1906-1918.

Cressie, N. A. C. (1993). Statistics for Spatial Data, Second edition. New York: Wiley.

Diggle, P. J. \& Hutchinson, M. F. (1989). On spline smoothing with correlated errors. Aust. J. Statist. 31, $166-182$.

FAn, J. \& GiJbels, I. (1996). Local Polynomial Modelling and Its Applications. New York: Chapman \& Hall/CRC.

Francisco-Fernández, M.\& Vilar-Fernández, J. M. (2001). Polynomial regression with correlated errors. Comm. Statist. Theory Methods, 30, 1271-1293.

Francisco-Fernández, M., Opsomer, J. D. \& Vilar-Fernández, J. M. (2004). Plug-in bandwidth selector for local polynomial regression estimator with correlated errors. J. Nonparametr. Stat., 16, 127-151.

Gijbels, I., Pope, A. \& WAnd, M.P. (1999). Understanding esponential smoothing via kernel regression. J. $R$. Statist. Soc. B 61, 39-50.

Hall, P., LAhiRi, S.N. \& Polzehl, J. (1995). On bandwidth choice in nonparametric regression with both shortand long-range dependent errors Ann. Statist. 23, 1921-1936.

265 Hall, P. \& VAN Keilegom, I. (2003). Using difference-based methods for inference in nonparametric regression with time series errors J. R. Statist. Soc. B. 65, 443456.

HART, J. D. (1991). Kernel regression estimation with time series errors. J. R. Statist. Soc. B. 53, 173-187.

Konishi, S. \& Kitagawa, G. (2008). Information Criteria and Statistical Modeling. New York: Springer.

Kim, T.Y., Kim, D., PARK, B.U. \& Simpson D.G. (2004). Nonparametric detection of correlated errors. Biometrika 91, 491-496.

Kim, T.Y., PARK B.U., Moon M.S. \& Kim C. (2009). Using bimodal kernel for inference in nonparametric regression with correlated errors. J. Multivariate Anal. 100, 1487-1497.

LeE, K.L., MAMmen, E. \& PARK, B.U. (2010). Bandwidth selection for kernel regression with correlated errors Statistics 44, 327-340.

LI, C.K. (1987). Asymptotic optimality for $C_{p}, C_{L}$, cross-validation and generalized cross-Validation: Discrete Index Set Ann. Statist. 15, 958-975. 
J. Opsomer, Y. WANG \& Y. YAng (2001). Nonparametric regression with correlated errors. Statist. Sci., 16, 134-153.

PARK, B.U., LEE, Y.K., KIM, T.Y. \& PARK, C. (2006). A simple estimator of error correlation in non-parametric regression models. Scand. J. Stat. 33, 451-462.

RUPPERT, D. \& WAND, M.P. (1994). Multivariate locally weighted least squares regression Ann. Statist. 22, 13461370 . 


\title{
Supplementary material for the paper: Local Polynomial Regression with Correlated Errors in Random Design and Unknown Correlation Structure
}

\author{
By K. DE BRABANTER AND F. CAO \\ Department of Statistics, Iowa State University, Ames, Iowa 50011, U.S.A. \\ kbrabant@iastate.edu fancao@iastate.edu \\ AND I. GIJBELS \\ Department of Mathematics, Katholieke Universiteit Leuven, Celestijnenlaan 200B, B-3001 \\ Leuven, Belgium \\ irene.gijbels@wis.kuleuven.be \\ AND J. OPSOMER \\ Department of Statistics, Colorado State University, Fort Collins, Colorado 80523, U.S.A. \\ jopsomer@stat.colostate.edu

\section{PROOF OF THEOREM 1} \\ In order to prove Theorem 1, we need the following technical lemma. \\ Lemma A. Assume that $K$ is Lipschitz continuous, bounded and that Assumptions 7 and 8 \\ hold. Further assume that $\lim _{n \rightarrow \infty} n \int \rho_{n}(x) d x=\rho_{c}, h \rightarrow 0$ and $n h \rightarrow \infty$ as $n \rightarrow \infty$ then

$$
\lim _{n \rightarrow \infty} n h \iint u^{s} K(u) v^{l} K(v) \rho_{n}\{h(u-v)\} d u d v=\rho_{c} \nu_{s+l}
$$

with $\nu_{j}=\int u^{j} K^{2}(u) d u$ and $0 \leq s, l \leq p$.

Proof of Lemma A. Let $g_{n}(v)=n h \int u^{s} K(u) \rho_{n}\{h(u-v)\} d u$. Then it immediately follows that

$$
n h \iint u^{s} K(u) v^{l} K(v) \rho_{n}\{h(u-v)\} d u d v=\int v^{l} K(v) g_{n}(v) d v .
$$

Consider the decomposition of $g_{n}(v)$ :

$$
\begin{aligned}
g_{n}(v)= & n h v^{s} K(v) \int_{|u-v|<\epsilon} \rho_{n}\{h(u-v)\} d u \\
& +n h \int_{|u-v|<\epsilon}\left\{u^{s} K(u)-v^{s} K(v)\right\} \rho_{n}\{h(u-v)\} d u \\
& +n h \int_{|u-v| \geq \epsilon} u^{s} K(u) \rho_{n}\{h(u-v)\} d u \\
= & I_{1}+I_{2}+I_{3} .
\end{aligned}
$$


The first term

$$
\begin{aligned}
I_{1} & =n h v^{s} K(v) \int_{|u-v|<\epsilon} \rho_{n}\{h(u-v)\} d u \\
& =v^{s} K(v) n \int_{\left|\frac{t}{h}\right|<\epsilon} \rho_{n}(t) d t \\
& =v^{s} K(v)\left\{n \int \rho_{n}(t) d t-n \int_{\left|\frac{t}{h}\right| \geq \epsilon} \rho_{n}(t) d t\right\} \\
& =v^{s} K(v)\left\{n \int \rho_{n}(t) d t-n \int_{|t| \geq \epsilon} \rho_{n}(h t) d t\right\} .
\end{aligned}
$$

As shown in a 2001 Iowa State University Department of Statistics PhD thesis by X.-H. Liu the last term goes to zero when $n \rightarrow \infty$ and hence by assumption of the lemma

$$
\lim _{n \rightarrow \infty} I_{1}=v^{s} K(v) \lim _{n \rightarrow \infty} n \int \rho_{n}(t) d t=v^{s} K(v) \rho_{c} .
$$

For the second term, we have that since $K$ is Lipschitz continuous it is differentiable almost everywhere. Also, $u^{s}, s=0, \ldots, p$ is differentiable implying $L(u)=u^{s} K(u)$ is differentiable almost everywhere. This yields

$$
\begin{aligned}
\left|I_{2}\right| & \leq n h \int_{|u-v|<\epsilon}\left|u^{s} K(u)-v^{s} K(v)\right|\left|\rho_{n}\{h(u-v)\}\right| d u \\
& =n h \int_{|u-v|<\epsilon}\left|v^{s} K(v)+L^{\prime}(v)(u-v)+O\left\{(u-v)^{2}\right\}-v^{s} K(v)\right|\left|\rho_{n}\{h(u-v)\}\right| d u \\
& \leq\left\{\left|L^{\prime}(v)\right| \epsilon+O\left(\epsilon^{2}\right)\right\}\left\{n \int_{\left|\frac{t}{h}\right|<\epsilon}\left|\rho_{n}(t)\right| d t\right\} \\
& \leq\left\{\left|L^{\prime}(v)\right| \epsilon+O\left(\epsilon^{2}\right)\right\} \rho_{\max }, \quad \text { by Assumption 8. }
\end{aligned}
$$

Consequently $\left|I_{2}\right| \rightarrow 0$ since $\epsilon$ can be made arbitrary small and $L^{\prime}(\cdot)$ is essentially bounded in magnitude by the Lipschitz constant. Finally, for the third term

$$
\begin{aligned}
\left|I_{3}\right| & \leq n h \int_{|u-v| \geq \epsilon}\left|u^{s} K(u)\right|\left|\rho_{n}\{h(u-v)\}\right| d u \\
& \leq \operatorname{Cnh} \int_{|t| \geq \epsilon}\left|\rho_{n}(h t)\right| d u \quad \text { since } u^{s} K(u)<\infty .
\end{aligned}
$$

30

Again, as shown in a 2001 Iowa State University Department of Statistics PhD thesis by X.-H. Liu $\left|I_{3}\right|$ goes to zero when $n \rightarrow \infty$. Combining the above results, we have shown that

$$
\lim _{n \rightarrow \infty} g_{n}(v)=v^{s} K(v) \rho_{c} \text {. }
$$

Next, note that

$$
\begin{aligned}
\left|g_{n}(v)\right| & \leq n h \int\left|u^{s} K(u)\right|\left|\rho_{n}\{h(u-v)\}\right| d u \\
& \leq C n \int\left|\rho_{n}(t)\right| d t .
\end{aligned}
$$

By assumption $n \int\left|\rho_{n}(t)\right| d t$ is bounded and hence $g_{n}(v)$ is also bounded. Since $L(\cdot)$ and $\rho_{n}(\cdot)$ are continuous it follows that $u^{s} K(u) \rho_{n}\{h(u-v)\}$ is also continuous. This implies that $g_{n}(v)$ 
is also continuous. Therefore, applying Lebesgue's bounded convergence theorem to change the 35 order of the limit and the integral yields

$$
\lim _{n \rightarrow \infty} \int v^{l} K(v) g_{n}(v) d v=\int v^{l} K(v) \lim _{n \rightarrow \infty} g_{n}(v) d v=\int v^{l} K(v) v^{s} K(v) \rho_{c} d v=\rho_{c} \nu_{s+l},
$$

proving the lemma.

THEOREM 1. Assume $f(x)>0$, and let $f(\cdot)$ and $m^{(p+1)}(\cdot)$ be continuous in a neighborhood of $x$. Further assume $K$ is Lipschitz continuous and $\lim _{n \rightarrow \infty} n \int \rho_{n}(x) d x=\rho_{c}$. Then under Assumptions 3, 4, 5, 7, 8 and for $h \rightarrow 0$ and $n h \rightarrow \infty$, the asymptotic conditional bias and variance for the local polynomial regression estimator of odd order $p$ are

$$
\operatorname{bias}\left\{\widehat{m}(x) \mid X_{1}, \ldots, X_{n}\right\}=\varepsilon_{1}^{T} S^{-1} \frac{c_{p}}{(p+1) !} m^{(p+1)}(x) h^{p+1}+o_{p}\left(h^{p+1}\right)
$$

and

$$
\operatorname{var}\left\{\widehat{m}(x) \mid X_{1}, \ldots, X_{n}\right\}=\frac{\sigma^{2}\left\{1+f(x) \rho_{c}\right\}}{n h f(x)} \varepsilon_{1}^{T} S^{-1} S^{\star} S^{-1} \varepsilon_{1}+o_{p}\left(\frac{1}{n h}\right),
$$

where $S=\left(\mu_{i+j}\right)_{0 \leq i, j \leq p}$ with $\mu_{j}=\int u^{j} K(u) d u, S^{\star}=\left(\nu_{i+j}\right)_{0 \leq i, j \leq p}$ with $\nu_{j}=\int u^{j} K^{2}(u) d u$ and $c_{p}=\left(\mu_{p+1}, \ldots, \mu_{2 p+1}\right)^{T}$.

Proof of Theorem 1. The local polynomial estimator is $\widehat{\beta}=\left(X_{x}^{T} \mathrm{~W}_{x} X_{x}\right)^{-1} X_{x}^{T} \mathrm{~W}_{x} Y$. Then $\quad{ }_{45}$ the conditional variance of the estimator in a point $x$ can be written as

$$
\begin{aligned}
\operatorname{var}\left(\widehat{\beta} \mid X_{1}, \ldots, X_{n}\right) & =\left(\frac{1}{n} X_{x}^{T} \mathrm{~W}_{x} X_{x}\right)^{-1}\left(\frac{1}{n^{2}} X_{x}^{T} W_{x} V W_{x} X_{x}\right)\left(\frac{1}{n} X_{x}^{T} \mathrm{~W}_{x} X_{x}\right)^{-1} \\
& =S_{n}^{-1} S_{n}^{\star} S_{n}^{-1},
\end{aligned}
$$

where $V$ is the variance matrix of the vector of random errors. The main idea of the proof is to find asymptotic expressions for $S_{n}$ and $S_{n}^{\star}$. According to Fan \& Gijbels (1996)

$$
S_{n}=f(x) H S H\left\{1+o_{p}(1)\right\}
$$

with $H=\operatorname{diag}\left(1, h, \ldots, h^{p}\right), S=\left(\mu_{i+j}\right)_{0 \leq i, j \leq p}$ and $\mu_{l}=\int u^{l} K(u) d u$. It follows that

$$
S_{n,(s, l)}^{\star}=\frac{\sigma^{2}}{n^{2}} \sum_{i=1}^{n} \sum_{k=1}^{n} K_{h}\left(X_{i}-x\right) K_{h}\left(X_{k}-x\right) \rho_{n}\left(X_{i}-X_{k}\right)\left(X_{i}-x\right)^{s}\left(X_{k}-x\right)^{l}
$$

with $S_{n}^{\star}$ the $(p+1) \times(p+1)$ matrix $\left(S_{n,(s, l)}^{\star}\right)_{0 \leq s, l \leq p}$. We have that

$$
\begin{aligned}
S_{n,(s, l)}^{\star}= & \frac{\sigma^{2}}{n^{2}} \sum_{i=1}^{n} K_{h}^{2}\left(X_{i}-x\right)\left(X_{i}-x\right)^{s+l} \\
& +\frac{\sigma^{2}}{n^{2}} \sum_{i=1}^{n} \sum_{k \neq i}^{n} K_{h}\left(X_{i}-x\right) K_{h}\left(X_{k}-x\right) \rho_{n}\left(X_{i}-X_{k}\right)\left(X_{i}-x\right)^{s}\left(X_{k}-x\right)^{l} \\
= & I_{1}+I_{2} .
\end{aligned}
$$

The first term yields (Fan \& Gijbels, 1996)

$$
I_{1}=\frac{\sigma^{2} h^{s+l-1}}{n} f(x) \nu_{s+l}\left\{1+o_{p}(1)\right\} .
$$


Next we show that

$$
I_{2}=\frac{\sigma^{2} h^{s+l-1}}{n} f^{2}(x) \nu_{s+l} \rho_{c}\left\{1+o_{p}(1)\right\}
$$

Consequently, it is sufficient to show that

$$
\lim _{n \rightarrow \infty} E\left(n h^{1-s-l} I_{2}\right)=\sigma^{2} f^{2}(x) \nu_{s+l} \rho_{c}
$$

and

$$
\lim _{n \rightarrow \infty} \operatorname{var}\left(n h^{1-s-l} I_{2}\right)=0
$$

$$
\begin{aligned}
E\left(n h^{1-s-l} I_{2}\right) & =\frac{\sigma^{2} n\left(n^{2}-n\right) h^{1-s-l}}{n^{2} h^{2}} \iint K\left(\frac{\alpha-x}{h}\right) K\left(\frac{\beta-x}{h}\right) \rho_{n}(\alpha-\beta)(\alpha-x)^{s}(\beta-x)^{l} f(\alpha) f(\beta) d \alpha d \beta \\
& =\sigma^{2}\left(1-\frac{1}{n}\right) n h \iint u^{s} K(u) v^{l} K(v) \rho_{n}\{h(u-v)\} f(x+u h) f(x+v h) d u d v \\
& =\sigma^{2}\left(1-\frac{1}{n}\right) f^{2}(x)\left[n h \iint u^{s} K(u) v^{l} K(v) \rho_{n}\{h(u-v)\} d u d v\right]\{1+o(1)\}
\end{aligned}
$$

provided that $h \rightarrow 0$. Taking the limit and using Lemma A yields

$$
\lim _{n \rightarrow \infty} E\left(n h^{1-s-l} I_{2}\right)=\sigma^{2} f^{2}(x) \rho_{c} \nu_{s+l} .
$$

Next, we prove that (4) holds. We have

$$
\operatorname{var}\left(n h^{1-s-l} I_{2}\right)=\frac{4 \sigma^{4} h^{2(1-s-l)}}{n^{2}} \sum_{i=1}^{n-1} \sum_{k=i+1}^{n} \sum_{l=1}^{n-1} \sum_{m=l+1}^{n} \operatorname{cov}\left\{\theta_{i k}(x), \theta_{l m}(x)\right\}
$$

with $\theta_{i k}(x)=K_{h}\left(X_{i}-x\right) K_{h}\left(X_{k}-x\right) \rho_{n}\left(X_{i}-X_{k}\right)\left(X_{i}-x\right)^{s}\left(X_{k}-x\right)^{l}$. Consider the three possibilities: First, all $i, k, l, m$ are different. Then $\operatorname{cov}\left\{\theta_{i k}(x), \theta_{l m}(x)\right\}=0$ since $\theta_{i k}(x)$ and $\theta_{l m}(x)$ are independent. Second, one of the $i$ and $k$ is equal to one of the $l$ and $m$. The total number of terms is bounded above by $n^{3}$. Note that $K$ and $\rho_{n}$ are symmetric and then for $i=l$ and $k \neq m$ we have

$$
\begin{aligned}
& \operatorname{cov}\left\{\theta_{i k}(x), \theta_{i m}(x)\right\} \\
= & E\left\{\theta_{i k}(x) \theta_{i m}(x)\right\}-E\left\{\theta_{i k}(x)\right\} E\left\{\theta_{i m}(x)\right\} \\
\leq & E\left\{\theta_{i k}(x) \theta_{i m}(x)\right\} \\
= & E\left\{K_{h}\left(X_{i}-x\right) K_{h}\left(X_{k}-x\right) \rho_{n}\left(X_{i}-X_{k}\right)\left(X_{i}-x\right)^{s}\left(X_{k}-x\right)^{l}\right. \\
& \left.\times K_{h}\left(X_{i}-x\right) K_{h}\left(X_{m}-x\right) \rho_{n}\left(X_{i}-X_{m}\right)\left(X_{i}-x\right)^{s}\left(X_{m}-x\right)^{l}\right\} \\
= & \frac{1}{h^{4}} \iiint K^{2}\left(\frac{u-x}{h}\right) K\left(\frac{v-x}{h}\right) K\left(\frac{w-x}{h}\right) \rho_{n}\{h(u-v)\} \rho_{n}\{h(u-w)\}(u-x)^{2 s}(v-x)^{l}(w-x)^{l} \\
& \times f(u) f(v) f(w) d u d v d w .
\end{aligned}
$$


Note that there exist $U$ and $C$ such that $|u|>U$ implies $u^{l} K(u)<C$ for $l=0, \ldots, p$

$$
\begin{aligned}
& \operatorname{cov}\left\{\theta_{i k}(x), \theta_{i m}(x)\right\} \\
\leq & \frac{1}{h^{4}} \iiint\left|(u-x)^{s} K\left(\frac{u-x}{h}\right)\right|^{2}\left|(v-x)^{l} K\left(\frac{v-x}{h}\right)\right|\left|(w-x)^{l} K\left(\frac{w-x}{h}\right)\right| \\
& \times\left|\rho_{n}\{h(u-v)\}\right|\left|\rho_{n}\{h(u-w)\}\right| f(u) f(v) f(w) d u d v d w \\
\leq & \frac{h^{2(s+l)} f_{\max }^{3}}{n^{2} h^{3}}\left(U^{2 s} K_{\max }^{2}+C^{2}\right)\left(U^{l} K_{\max }+C\right)^{2}\left[n^{2} h^{2} \iiint\left|\rho_{n}\{h(u-v)\}\right|\left|\rho_{n}\{h(u-w)\}\right| d u d v d w\right] \\
= & \frac{h^{2(s+l)} f_{\max }^{3}}{n^{2} h^{3}} C_{1} n^{2} h^{2} \int\left[\int\left|\rho_{n}\{h(u-v)\}\right| d v\right]\left[\int\left|\rho_{n}\{h(u-w)\}\right| d w\right] d u \\
= & \frac{h^{2(s+l)} f_{\max }^{3}}{n^{2} h^{3}} C_{1} \int\left\{n \int\left|\rho_{n}(\alpha)\right| d \alpha\right\}\left\{n \int\left|\rho_{n}(\beta)\right| d \beta\right\} d u \\
\leq & \frac{h^{2(s+l)} f_{\max }^{3} C_{1} \rho_{\max }^{2} \mu(\Omega)}{n^{2} h^{3}}=\frac{C_{2}}{n^{2} h^{3-2 s-2 l}}, \quad \text { by Assumption 8. }
\end{aligned}
$$

Third and last case is for $i=l<k=m$. The total number of terms is $\sum_{a=1}^{n-1}(n-a)=\left(n^{2}-\right.$ $n) / 2$. Using similar arguments as before yields

$$
\begin{aligned}
\operatorname{cov}\left\{\theta_{i k}(x), \theta_{i k}(x)\right\} & =\operatorname{var}\left\{K_{h}\left(X_{i}-x\right) K_{h}\left(X_{k}-x\right) \rho_{n}\left(X_{i}-X_{k}\right)\left(X_{i}-x\right)^{s}\left(X_{k}-x\right)^{l}\right\} \\
& \leq E\left\{K_{h}^{2}\left(X_{i}-x\right) K_{h}^{2}\left(X_{k}-x\right) \rho_{n}^{2}\left(X_{i}-X_{k}\right)\left(X_{i}-x\right)^{2 s}\left(X_{k}-x\right)^{2 l}\right\} \\
& \leq \frac{C_{3} f_{\max }^{2}}{n h^{3-2 s-2 l}} \int\left[n h \int \rho_{n}^{2}\{h(\alpha-\beta)\} d \alpha\right] d \beta .
\end{aligned}
$$

Since $\left|\rho_{n}(x)\right| \leq 1$ for all $x$

$$
n h \int \rho_{n}^{2}\{h(\alpha-\beta)\} d \alpha \leq n \int\left|\rho_{n}(t)\right| d t \leq \rho_{\max }
$$

and consequently

$$
\operatorname{cov}\left\{\theta_{i k}(x), \theta_{i k}(x)\right\} \leq \frac{C_{3} f_{\max }^{2}}{n h^{3-2 s-2 l}} \rho_{\max } \mu(\Omega)=\frac{C_{4}}{n h^{3-2 s-2 l}} .
$$

Combining the three results yields

$$
\begin{aligned}
\operatorname{var}\left(n h^{1-s-l} I_{2}\right) & \leq \frac{4 \sigma^{4} h^{2(1-s-l)}}{n^{2}}\left(n^{3} \frac{C_{2}}{n^{2} h^{3-2 s-2 l}}+\frac{n^{2}-n}{2} \frac{C_{4}}{n h^{3-2 s-2 l}}\right) \\
& =\frac{4 \sigma^{2}}{n h}\left\{C_{2}+C_{5}\left(1-n^{-1}\right)\right\} \\
& \rightarrow 0, \quad \text { as } n \rightarrow \infty,
\end{aligned}
$$

and hence (2) is established.

According to (1) and (2) the matrix $S_{n}^{\star}$ is given by

$$
S_{n}^{\star}=\frac{\sigma^{2}}{n h} f(x)\left\{1+f(x) \rho_{c}\right\} H S^{\star} H\left\{1+o_{p}(1)\right\} .
$$


The variance of the local polynomial regression estimator with correlated errors immediately follows

$$
\begin{aligned}
\operatorname{var}\left\{\widehat{m}(x) \mid X_{1}, \ldots, X_{n}\right\} & =\varepsilon_{1}^{T} \operatorname{var}\left(\widehat{\beta} \mid X_{1}, \ldots, X_{n}\right) \varepsilon_{1} \\
& =\frac{\sigma^{2}\left\{1+f(x) \rho_{c}\right\}}{n h f(x)} \varepsilon_{1}^{T} S^{-1} S^{\star} S^{-1} \varepsilon_{1}+o_{p}\left(\frac{1}{n h}\right) .
\end{aligned}
$$

\section{Proof of THEOREM 2}

THEOREM 2. Under Assumption 1 to 10

$$
\operatorname{RSS}(h)=\operatorname{ASE}(h)+\frac{1}{n} \sum_{i=1}^{n} e_{i}^{2}-\frac{2 \sigma^{2} K(0) S^{00}\left\{\mu(\Omega)+\rho_{c}\right\}}{n h}+o_{p}\left(n^{-1} h^{-1}\right),
$$

where $S^{00}$ denotes the first element of the first row of the inverse of the matrix $S$.

Proof of Theorem 2. Consider the decomposition

$$
\begin{aligned}
\operatorname{RSS}(h)-\operatorname{ASE}(h)= & \frac{1}{n} \sum_{i=1}^{n}\left\{Y_{i}-\widehat{m}\left(X_{i}\right)\right\}^{2}-\frac{1}{n} \sum_{i=1}^{n}\left\{m\left(X_{i}\right)-\widehat{m}\left(X_{i}\right)\right\}^{2} \\
= & \frac{1}{n} \sum_{i=1}^{n} e_{i}^{2}+\frac{2}{n} \sum_{i=1}^{n}\left\{m\left(X_{i}\right)-\widehat{m}\left(X_{i}\right)\right\} e_{i} \\
= & \frac{1}{n} \sum_{i=1}^{n} e_{i}^{2}+\frac{2}{n} \sum_{i=1}^{n}\left\{m\left(X_{i}\right)-\sum_{j=1}^{n} W_{j}^{n}\left(X_{i}\right) m\left(X_{j}\right)\right\} e_{i}-\frac{2}{n} \sum_{i=1}^{n} \sum_{j=1}^{n} W_{j}^{n}\left(X_{i}\right) e_{i} e_{j} \\
= & \frac{1}{n} \sum_{i=1}^{n} e_{i}^{2}+\frac{2}{n} \sum_{i=1}^{n}\left\{m\left(X_{i}\right)-\sum_{j=1}^{n} W_{j}^{n}\left(X_{i}\right) m\left(X_{j}\right)\right\} e_{i}-\frac{2}{n} \sum_{i=1}^{n} W_{i}^{n}\left(X_{i}\right) e_{i}^{2} \\
& -\frac{2}{n} \sum_{i=1}^{n} \sum_{j \neq i}^{n} W_{j}^{n}\left(X_{i}\right) e_{i} e_{j} \\
= & \frac{1}{n} \sum_{i=1}^{n} e_{i}^{2}+I_{1}-I_{2}-I_{3} .
\end{aligned}
$$

For $I_{1}$ we have

$$
E\left[\frac{2}{n} \sum_{i=1}^{n}\left\{m\left(X_{i}\right)-\sum_{j=1}^{n} W_{j}^{n}\left(X_{i}\right) m\left(X_{j}\right)\right\} e_{i} \mid X_{1}, \ldots, X_{n}\right]=0
$$

and

$$
\begin{aligned}
& \operatorname{var}\left[\frac{2}{n} \sum_{i=1}^{n}\left\{m\left(X_{i}\right)-\sum_{j=1}^{n} W_{j}^{n}\left(X_{i}\right) m\left(X_{j}\right)\right\} e_{i} \mid X_{1}, \ldots, X_{n}\right] \\
= & \frac{4}{n^{2}} \sum_{i=1}^{n} \sum_{j=1}^{n} \operatorname{cov}\left[\operatorname{bias}\left\{\widehat{m}\left(X_{i}\right) \mid X_{1}, \ldots, X_{n}\right\} e_{i}, \operatorname{bias}\left\{\widehat{m}\left(X_{j}\right) \mid X_{1}, \ldots, X_{n}\right\} e_{j} \mid X_{1}, \ldots, X_{n}\right] \\
= & I_{1 a}+I_{1 b},
\end{aligned}
$$


with

$$
I_{1 a}=\frac{4 \sigma^{2}}{n^{2}} \sum_{i=1}^{n} \operatorname{bias}^{2}\left\{\widehat{m}\left(X_{i}\right) \mid X_{1}, \ldots, X_{n}\right\}=O_{p}\left(\frac{h^{2 p+2}}{n}\right)
$$

and

$$
I_{1 b}=\frac{4}{n^{2}} \sum_{i=1}^{n} \sum_{j \neq i}^{n} \operatorname{bias}\left\{\widehat{m}\left(X_{i}\right) \mid X_{1}, \ldots, X_{n}\right\} \operatorname{bias}\left\{\widehat{m}\left(X_{j}\right) \mid X_{1}, \ldots, X_{n}\right\} \operatorname{cov}\left(e_{i}, e_{j} \mid X_{i}, X_{j}\right) .
$$

In what follows, the main ideas are using Riemann approximations of sums by integrals, change of variables, considering the tail conditions on the kernel $K$ and the assumptions on the correlation function and the density. The term $I_{1 b}$ yields

$$
\begin{aligned}
\left|I_{1 b}\right| & \leq 4 \sigma^{2} O_{p}\left(h^{2 p+2}\right) \frac{1}{n^{2}} \sum_{i=1}^{n} \sum_{j \neq i}^{n}\left|\rho_{n}\left(X_{i}-X_{j}\right)\right| \\
& =4 \sigma^{2} O_{p}\left(h^{2 p+2}\right) \iint\left|\rho_{n}(u-v)\right| f(u) f(v) d u d v\left\{1+o_{p}(1)\right\} \\
& \leq \frac{4 \sigma^{2} f_{\max }^{2}}{n} O_{p}\left(h^{2 p+2}\right) \int\left\{n \int\left|\rho_{n}(t)\right| d t\right\} d v\left\{1+o_{p}(1)\right\} \\
& =O_{p}\left(\frac{h^{2 p+2}}{n}\right) .
\end{aligned}
$$

Combining the above results gives

$$
I_{1}=O_{p}\left(\frac{h^{p+1}}{\sqrt{n}}\right)
$$

Next, we show that

$$
I_{2}=\frac{2 \sigma^{2} S^{00} K(0) \mu(\Omega)}{n h}\left\{1+o_{p}(1)\right\}
$$

Hence it is sufficient to show that

$$
\lim _{n \rightarrow \infty} E\left(n h I_{2} \mid X_{1}, \ldots, X_{n}\right)=2 \sigma^{2} S^{00} K(0) \mu(\Omega) \quad \text { and } \quad \lim _{n \rightarrow \infty} \operatorname{var}\left(n h I_{2} \mid X_{1}, \ldots, X_{n}\right)=0 .
$$

Using the equivalent kernel expression in Fan \& Gijbels (1996, p. 64), the law of large numbers and the fact that the density has bounded support yields

$$
\begin{aligned}
E\left(n h I_{2} \mid X_{1}, \ldots, X_{n}\right) & =2 h \sigma^{2} \sum_{i=1}^{n} \frac{1}{n h f\left(X_{i}\right)} S^{00} K(0) \\
& =2 \sigma^{2} S^{00} K(0) \frac{1}{n} \sum_{i=1}^{n} \frac{1}{f\left(X_{i}\right)}
\end{aligned}
$$

and consequently

$$
\lim _{n \rightarrow \infty} E\left(n h I_{2} \mid X_{1}, \ldots, X_{n}\right)=2 \sigma^{2} S^{00} K(0) \mu(\Omega)
$$


90

$$
\begin{aligned}
\operatorname{var}\left(n h I_{2} \mid X_{1}, \ldots, X_{n}\right) & =n^{2} h^{2} \frac{4}{n^{2}} \sum_{i=1}^{n} \sum_{j=1}^{n} W_{i}^{n}\left(X_{i}\right) W_{j}^{n}\left(X_{j}\right) \operatorname{cov}\left(e_{i}^{2}, e_{j}^{2} \mid X_{i}, X_{j}\right) \\
& =I_{2 a}+I_{2 b}
\end{aligned}
$$

with

$$
I_{2 a}=4 h^{2} \sum_{i=1}^{n}\left\{W_{i}^{n}\left(X_{i}\right)\right\}^{2} \operatorname{cov}\left(e_{i}^{2}, e_{i}^{2} \mid X_{i}, X_{j}\right)
$$

and

$$
I_{2 b}=4 h^{2} \sum_{i=1}^{n} \sum_{j \neq i}^{n} W_{i}^{n}\left(X_{i}\right) W_{j}^{n}\left(X_{j}\right) \operatorname{cov}\left(e_{i}^{2}, e_{j}^{2} \mid X_{i}, X_{j}\right) .
$$

Using the assumption

$$
\begin{aligned}
\operatorname{cov}\left(e_{i} e_{j}, e_{k} e_{l} \mid X_{i}, X_{j}, X_{k}, X_{l}\right)= & \operatorname{cov}\left(e_{i}, e_{k} \mid X_{i}, X_{k}\right) \operatorname{cov}\left(e_{j}, e_{l} \mid X_{j}, X_{l}\right) \\
& +\operatorname{cov}\left(e_{i}, e_{l} \mid X_{i}, X_{l}\right) \operatorname{cov}\left(e_{j}, e_{k} \mid X_{j}, X_{k}\right)
\end{aligned}
$$

for all $i, j, k, l$ and under the assumptions on the density, we have

$$
\begin{aligned}
I_{2 a} & =8 h^{2} \sum_{i=1}^{n} \frac{\operatorname{cov}^{2}\left(e_{i}, e_{i} \mid X_{i}, X_{j}\right)}{n^{2} h^{2} f^{2}\left(X_{i}\right)}\left(S^{00}\right)^{2} K^{2}(0)\left\{1+o_{p}(1)\right\} \\
& =\frac{8 \sigma^{4}\left(S^{00}\right)^{2} K^{2}(0)}{n} \int \frac{1}{f(x)} d x\left\{1+o_{p}(1)\right\} \\
& \rightarrow 0, \quad \text { for } n \rightarrow \infty
\end{aligned}
$$

and

$$
\begin{aligned}
I_{2 b} & =8 h^{2} O_{p}\left(\frac{1}{n^{2} h^{2}}\right) \sum_{i=1}^{n} \sum_{j \neq i}^{n} \operatorname{cov}^{2}\left(e_{i}, e_{j} \mid X_{i}, X_{j}\right) \\
& =8 \sigma^{4} O_{p}(1) \iint \rho_{n}^{2}(u-v) f(u) f(v) d u d v\left\{1+o_{p}(1)\right\} \\
& =8 O_{p}\left(\frac{1}{n}\right) \int\left\{n \int \rho_{n}^{2}(t) d t\right\} d v\left\{1+o_{p}(1)\right\} \\
& =O_{p}\left(\frac{1}{n}\right)
\end{aligned}
$$

where the last step follows from Assumptions 4 and 9. Taking the limit for $n \rightarrow \infty$ yields $I_{2 b}=$ $o_{p}(1)$. Combining these results gives

$$
\lim _{n \rightarrow \infty} \operatorname{var}\left(n h I_{2} \mid X_{1}, \ldots, X_{n}\right)=0 .
$$


For the third term and using the equivalent kernel expression in Fan \& Gijbels (1996, p. 64)

$$
\begin{aligned}
E\left(I_{3} \mid X_{1}, \ldots, X_{n}\right)= & \frac{2 \sigma^{2}}{n^{2} h} \sum_{i=1}^{n} \sum_{j \neq i}^{n} \frac{1}{f\left(X_{i}\right)}\left\{\sum_{l=0}^{p} S^{0 l}\left(\frac{X_{j}-X_{i}}{h}\right)^{l}\right\} K\left(\frac{X_{j}-X_{i}}{h}\right) \rho_{n}\left(X_{i}-X_{j}\right) \\
= & \frac{2 \sigma^{2}}{n^{2} h} \sum_{i=1}^{n} \sum_{j \neq i}^{n} \frac{1}{f\left(X_{i}\right)} S^{00} K\left(\frac{X_{j}-X_{i}}{h}\right) \rho_{n}\left(X_{i}-X_{j}\right) \\
& +\frac{2 \sigma^{2}}{n^{2} h} \sum_{i=1}^{n} \sum_{j \neq i}^{n} \frac{1}{f\left(X_{i}\right)}\left\{\sum_{l=1}^{p} S^{0 l}\left(\frac{X_{j}-X_{i}}{h}\right)^{l}\right\} K\left(\frac{X_{j}-X_{i}}{h}\right) \rho_{n}\left(X_{i}-X_{j}\right) \\
= & 2 \sigma^{2} S^{00} \iint \frac{1}{h} K\left(\frac{u-v}{h}\right) \rho_{n}(u-v) f(u) d u d v\left\{1+o_{p}(1)\right\} \\
& +2 \sigma^{2} \sum_{l=1}^{p} S^{0 l} \iint \frac{1}{h}\left(\frac{u-v}{h}\right)^{l} K\left(\frac{u-v}{h}\right) \rho_{n}(u-v) f(u) d u d v\left[1+o_{p}(1)\right] \\
= & I_{3 a}\left\{1+o_{p}(1)\right\}+I_{3 b}\left\{1+o_{p}(1)\right\} .
\end{aligned}
$$

Similarly to Lemma A it can be shown that

$$
\lim _{n \rightarrow \infty} n h \iint \frac{1}{h} K\left(\frac{u-v}{h}\right) \rho_{n}(u-v) f(u) d u d v=K(0) \rho_{c} .
$$

It follows that

$$
I_{3 a}=\frac{2 \sigma^{2} S^{00} K(0) \rho_{c}}{n h}\{1+o(1)\}
$$

Next, we bound the term $I_{3 b}$.

$$
I_{3 b}=\frac{1}{h} \iint\left(\frac{u-v}{h}\right)^{l} K\left(\frac{u-v}{h}\right) \rho_{n}(u-v) f(u) d u d v .
$$

Let $g_{n}(v)=\frac{1}{h} \int\left(\frac{u-v}{h}\right)^{l} K\left(\frac{u-v}{h}\right) \rho_{n}(u-v) f(u) d u$. Since the kernel $K$ is Lipschitz continuous and bounded, the density $f$ is bounded and by Assumption 7 there exist a $U$ and $C$ such that $|u|>U$ implies $|u|^{l} K(u)<C$ for $l=1, \ldots, p$

$$
\begin{aligned}
\left|g_{n}(v)\right| \leq & \int\left|t^{l} K(t)\right|\left|\rho_{n}(h t)\right| f(v+h t) d t \\
= & \int_{|t|<\varepsilon}\left|t^{l} K(t)\right|\left|\rho_{n}(h t)\right| f(v+h t) d t+\int_{|t| \geq \varepsilon}\left|t^{l} K(t)\right|\left|\rho_{n}(h t)\right| f(v+h t) d t \\
\leq & \frac{\varepsilon^{l}\{|K(0)+L \varepsilon|\} f_{\max } \rho_{\max }}{n h}+\int_{\varepsilon \leq|t| \leq U}\left|t^{l} K(t)\right|\left|\rho_{n}(h t)\right| f(v+h t) d t \\
& +\int_{|t|>U}\left|t^{l} K(t)\right|\left|\rho_{n}(h t)\right| f(v+h t) d t \\
\leq & \frac{\varepsilon^{l}|K(0)+L \varepsilon| f_{\max } \rho_{\max }}{n h}+\frac{U^{l} K_{\max } f_{\max }}{h} o\left(n^{-1}\right)+\frac{C f_{\max }}{h} o\left(n^{-1}\right)
\end{aligned}
$$


where the last step uses Assumption 8. Finally, combining all the steps together results in the upperbound

$$
\left|I_{3 b}\right| \leq \int\left\{\frac{\varepsilon^{l}|K(0)+L \varepsilon| f_{\max } \rho_{\max }}{n h}+\frac{U^{l} K_{\max } f_{\max }}{h} o\left(n^{-1}\right)+\frac{C f_{\max }}{h} o\left(n^{-1}\right)\right\} d v,
$$

and since $\varepsilon$ can be made arbitrary small and $l \neq 0$

$$
\left|I_{3 b}\right|=o\left(n^{-1} h^{-1}\right)
$$

This leads to the result

$$
\lim _{n \rightarrow \infty} E\left(n h I_{3} \mid X_{1}, \ldots, X_{n}\right)=2 \sigma^{2} S^{00} K(0) \rho_{c}
$$

The variance of $I_{3}$ is

$\operatorname{var}\left(I_{3} \mid X_{1}, \ldots, X_{n}\right)=\frac{4}{n^{2}} \sum_{i=1}^{n-1} \sum_{j=i+1}^{n} \sum_{k=1}^{n-1} \sum_{l=k+1}^{n} W_{j}^{n}\left(X_{i}\right) W_{k}^{n}\left(X_{l}\right) \operatorname{cov}\left(e_{i} e_{j}, e_{k} e_{l} \mid X_{i}, X_{j} X_{k}, X_{l}\right)$

We can distinguish three cases: First, $i=k$ and $j=l$ and the total number of terms is bounded above by $n^{2}$. Second, one of the $i$ and $j$ is equal to one of the $k$ and $l$. Without loss of generality, assume $i=k$ and $j \neq l$. The total terms can be bounded above by $n^{3}$. Finally, $i, k, l, m$ are all different.

For the first case and using Assumption 10,

$$
\begin{aligned}
I_{3 c} & =\frac{4}{n^{2}} \sum_{i=1}^{n-1} \sum_{j=i+1}^{n} W_{j}^{n}\left(X_{i}\right) W_{i}^{n}\left(X_{j}\right)\left\{\sigma^{4}+\operatorname{cov}^{2}\left(e_{i}, e_{j} \mid X_{i}, X_{j}\right)\right\} \\
& =I_{3 c}^{\prime}+I_{3 c}^{\prime \prime}
\end{aligned}
$$

where

$$
I_{3 c}^{\prime}=\frac{4 \sigma^{4}}{n^{2}} \sum_{i=1}^{n-1} \sum_{j=i+1}^{n} W_{j}^{n}\left(X_{i}\right) W_{i}^{n}\left(X_{j}\right)
$$

and

$$
I_{3 c}^{\prime \prime}=\frac{4 \sigma^{4}}{n^{2}} \sum_{i=1}^{n-1} \sum_{j=i+1}^{n} W_{j}^{n}\left(X_{i}\right) W_{i}^{n}\left(X_{j}\right) \rho_{n}^{2}\left(X_{i}-X_{j}\right)
$$


We have that using Riemann approximations of sums by integrals

$$
\begin{aligned}
I_{3 c}^{\prime}= & \frac{4 \sigma^{4}}{n^{2} h^{2}} \sum_{q=0}^{p} \sum_{w=0}^{p} S^{0 q} S^{0 w} \iint\left(\frac{v-u}{h}\right)^{q}\left(\frac{u-v}{h}\right)^{w} K^{2}\left(\frac{u-v}{h}\right) d u d v\left\{1+o_{p}(1)\right\} \\
= & \frac{4 \sigma^{4}}{n^{2} h^{2}} S^{00} S^{00} \iint K^{2}\left(\frac{u-v}{h}\right) d u d v\left\{1+o_{p}(1)\right\} \\
& +\frac{4 \sigma^{4}}{n^{2} h^{2}} \sum_{w=1}^{p} S^{00} S^{0 w} \iint\left(\frac{u-v}{h}\right)^{w} K^{2}\left(\frac{u-v}{h}\right) d u d v\left\{1+o_{p}(1)\right\} \\
& +\frac{4 \sigma^{4}}{n^{2} h^{2}} \sum_{q=1}^{p} S^{0 q} S^{00} \iint\left(\frac{v-u}{h}\right)^{q} K^{2}\left(\frac{u-v}{h}\right) d u d v\left\{1+o_{p}(1)\right\} \\
& +\frac{4 \sigma^{4}}{n^{2} h^{2}} \sum_{q=1}^{p} \sum_{w=1}^{p} S^{0 q} S^{0 w} \iint\left(\frac{v-u}{h}\right)^{q}\left(\frac{u-v}{h}\right)^{w} K^{2}\left(\frac{u-v}{h}\right) d u d v\left\{1+o_{p}(1)\right\} .
\end{aligned}
$$

By a change of variables, considering the assumptions on the kernel $K$ and the density $f$, the first term yields

$$
\begin{aligned}
\frac{4 \sigma^{4}}{n^{2} h^{2}} S^{00} S^{00} \iint K^{2}\left(\frac{u-v}{h}\right) d u d v & =\frac{4 \sigma^{4}}{n^{2} h} S^{00} S^{00} \int\left\{\int K^{2}(t) d t\right\} d v \\
& =O\left(\frac{1}{n^{2} h}\right)
\end{aligned}
$$

since the inner integral is bounded by Assumptions 6 and 7. This follows by using a similar 120 technique to bound (7). For the second term we have

$$
\begin{aligned}
\frac{4 \sigma^{4}}{n^{2} h^{2}} \sum_{w=0}^{p} S^{00} S^{0 w} \iint\left(\frac{u-v}{h}\right)^{w} K^{2}\left(\frac{u-v}{h}\right) d u d v & =\frac{4 \sigma^{4} S^{00}}{n^{2} h} \sum_{w=0}^{p} S^{0 w} \int\left\{\int t^{w} K^{2}(t) d t\right\} d v \\
& =O\left(\frac{1}{n^{2} h}\right)
\end{aligned}
$$

since the inner integral is bounded by Assumptions 6 and 7. Similarly, the third and fourth term can be shown to be $O_{p}\left(n^{-2} h^{-1}\right)$. The term $I_{3 c}^{\prime \prime}$ yields

$I_{3 c}^{\prime \prime}=\frac{4 \sigma^{4}}{n^{2} h^{2}} \sum_{q=0}^{p} \sum_{w=0}^{p} S^{0 q} S^{0 w} \iint\left(\frac{v-u}{h}\right)^{q}\left(\frac{u-v}{h}\right)^{w} K^{2}\left(\frac{u-v}{h}\right) \rho_{n}^{2}(u-v) d u d v\left\{1+o_{p}(1)\right\}$

Splitting this up in four terms as before i.e., for $q=w=0 ; q=0, w=1, \ldots, p ; w=0, q=$ $1, \ldots, p ; q=1, \ldots, p$ and $w=1, \ldots, p$ and under assumptions on the kernel, density and correlation function all terms can be shown to be $O_{p}\left(n^{-3} h^{-2}\right)$. We will show this for the first case. The other cases can be bounded using similar techniques as used in (7). The first term is given 
by

$$
\begin{aligned}
\frac{4 \sigma^{4}}{n^{2} h^{2}} S^{00} S^{00} \iint K^{2}\left(\frac{u-v}{h}\right) \rho_{n}^{2}(u-v) d u d v & \leq \frac{4 \sigma^{4}}{n^{2} h} S^{00} S^{00} K_{\max }^{2} \int\left\{\int \rho_{n}^{2}(h t) d t\right\} d v \\
& =\frac{4 \sigma^{4}}{n^{3} h^{2}} S^{00} S^{00} K_{\max }^{2} \int\left\{n \int \rho_{n}^{2}(u) d u\right\} d v \\
& =O\left(\frac{1}{n^{3} h^{2}}\right) .
\end{aligned}
$$

For the second case $(i=k$ and $j \neq l)$ and by Assumption 10 we have

$$
\begin{aligned}
I_{3 d} & =\frac{4 \sigma^{4}}{n^{2}} \sum_{i=1}^{n-1} \sum_{j=i+1}^{n} \sum_{l=i+2}^{n} W_{j}^{n}\left(X_{i}\right) W_{i}^{n}\left(X_{l}\right)\left\{\rho_{n}\left(X_{j}-X_{l}\right)+\rho_{n}\left(X_{i}-X_{l}\right) \rho_{n}\left(X_{j}-X_{i}\right)\right\} \\
& =I_{3 d}^{\prime}+I_{3 d}^{\prime \prime}
\end{aligned}
$$

The first term gives

$$
\begin{aligned}
I_{3 d}^{\prime}= & \frac{4 \sigma^{4}}{n^{2}} \sum_{i=1}^{n-1} \sum_{j=i+1}^{n} \sum_{l=i+2}^{n} W_{j}^{n}\left(X_{i}\right) W_{i}^{n}\left(X_{l}\right) \rho_{n}\left(X_{j}-X_{l}\right) \\
= & \frac{4 \sigma^{4}}{n h^{2}} S^{00} S^{00} \iiint K\left(\frac{v-u}{h}\right) K\left(\frac{t-u}{h}\right) \rho_{n}(v-t) f(v) d u d v d t\left\{1+o_{p}(1)\right\} \\
& +\frac{4 \sigma^{4} S^{00}}{n h^{2}} \sum_{w=1}^{p} S^{0 w} \iiint\left(\frac{t-u}{h}\right)^{w} K\left(\frac{v-u}{h}\right) K\left(\frac{t-u}{h}\right) \rho_{n}(v-t) f(v) d u d v d t\left\{1+o_{p}(1)\right\} \\
& +\frac{4 \sigma^{4} S^{00}}{n h^{2}} \sum_{q=1}^{p} S^{0 q} \iiint\left(\frac{v-u}{h}\right)^{q} K\left(\frac{v-u}{h}\right) K\left(\frac{t-u}{h}\right) \rho_{n}(v-t) f(v) d u d v d t\left\{1+o_{p}(1)\right\} \\
& +\frac{4 \sigma^{4}}{n h^{2}} \sum_{q=1}^{p} \sum_{w=1}^{p} S^{0 q} S^{0 w} \iiint\left(\frac{v-u}{h}\right)^{q} K\left(\frac{v-u}{h}\right)\left(\frac{t-u}{h}\right)^{w} K\left(\frac{t-u}{h}\right) \rho_{n}(v-t) f(v) d u d v d t\left\{1+o_{p}(1)\right\} \\
= & I_{3 d^{\prime}}^{\prime}\left\{1+o_{p}(1)\right\}+I_{3 d^{\prime \prime}}^{\prime}\left\{1+o_{p}(1)\right\}+I_{3 d^{\prime \prime \prime}}^{\prime}\left\{1+o_{p}(1)\right\}+I_{3 d^{\prime \prime \prime \prime}}^{\prime}\left\{1+o_{p}(1)\right\} .
\end{aligned}
$$

By a change of variables and Assumptions 4, 5 and 8 the term $I_{3 d^{\prime}}^{\prime}$ becomes

$$
\begin{aligned}
\left|I_{3 d^{\prime}}^{\prime}\right| & \leq \frac{4 \sigma^{4}\left(S^{00}\right)^{2} K_{\max }^{2} f_{\max }}{n} \iiint\left|\rho_{n}\{h(\alpha-\beta)\}\right| d \alpha d \beta d t \\
& =\frac{4 \sigma^{4}\left(S^{00}\right)^{2} K_{\max }^{2} f_{\max }}{n^{2} h} \iint\left\{n \int\left|\rho_{n}(u)\right| d u\right\} d \beta d t \\
& =O\left(\frac{1}{n^{2} h}\right) .
\end{aligned}
$$


Next, we will bound the term $I_{3 d^{\prime \prime \prime \prime}}^{\prime}$. The terms $I_{3 d^{\prime \prime}}^{\prime}$ and $I_{3 d^{\prime \prime \prime}}^{\prime}$ can be bounded in the same way and the proof is therefore omitted. Both terms $I_{3 d^{\prime \prime}}^{\prime}$ and $I_{3 d^{\prime \prime \prime}}^{\prime}$ are $o\left(n^{-1} h^{-1}\right)$.

$$
\begin{aligned}
\left|I_{3 d^{\prime \prime \prime \prime}}^{\prime}\right| & \leq \frac{4 \sigma^{4} f_{\max }}{n} \sum_{q=1}^{p} \sum_{w=1}^{p} S^{0 q} S^{0 w} \iiint\left|\alpha^{q} K(\alpha)\right|\left|\beta^{w} K(\beta)\right|\left|\rho_{n}(h(\alpha-\beta))\right| d \alpha d \beta d u \\
& =4 \sigma^{4} f_{\max } \sum_{q=1}^{p} \sum_{w=1}^{p} S^{0 q} S^{0 w} \iint g_{n}(\beta)\left|\beta^{w} K(\beta)\right| d \beta d u,
\end{aligned}
$$

with

$$
g_{n}(\beta)=\frac{1}{n} \int\left|\alpha^{q} K(\alpha)\right|\left|\rho_{n}\{h(\alpha-\beta)\}\right| d \alpha .
$$

The kernel is Lipschitz continuous and bounded; by Assumption 7 there exist a $U$ and $C$ such $\quad{ }_{135}$ that $|u|>U$ implies $|u|^{q} K(u)<C$ for $q=1, \ldots, p$. It follows that

$$
\begin{aligned}
g_{n}(\beta) & =\frac{1}{n} \int_{|\alpha| \leq \epsilon}\left|\alpha^{q} K(\alpha)\right|\left|\rho_{n}\{h(\alpha-\beta)\}\right| d \alpha+\frac{1}{n} \int_{|\alpha|>\epsilon}\left|\alpha^{q} K(\alpha)\right|\left|\rho_{n}\{h(\alpha-\beta)\}\right| d \alpha \\
& \leq \frac{\epsilon^{q}\{|K(0)+L \epsilon|\} \rho_{\max }}{n h}+\frac{U K_{\max }}{h} \int_{\epsilon<|\alpha| \leq U}\left|\rho_{n}(t)\right| d t+\frac{C}{h} \int_{|\alpha|>U}\left|\rho_{n}(t)\right| d t \\
& \leq \frac{\epsilon^{q}\{|K(0)+L \epsilon|\} \rho_{\max }}{n h}+\frac{U K_{\max }}{h} o\left(n^{-1}\right)+\frac{C}{h} o\left(n^{-1}\right),
\end{aligned}
$$

by Assumption 8. Since $\epsilon$ can be made arbitrary small and $q \neq 0$ we have $g_{n}(\beta)=o\left(n^{-1} h^{-1}\right)$. Consequently, the term $I_{3 d^{\prime \prime \prime \prime}}^{\prime}$ is bounded by

$$
\begin{aligned}
\left|I_{3 d^{\prime \prime \prime}}^{\prime \prime}\right| & \leq 4 \sigma^{4} f_{\max } \sum_{q=1}^{p} \sum_{w=1}^{p} S^{0 q} S^{0 w} o\left(n^{-1} h^{-1}\right) \int\left\{\int\left|\beta^{w} K(\beta)\right| d \beta\right\} d u \\
& =o\left(\frac{1}{n h}\right)
\end{aligned}
$$

due to the assumptions on the kernel and the density. For the last case (when all $i, j, k, l$ are different), we have by Assumption 10

$$
\begin{aligned}
I_{3 e}= & \frac{4}{n^{2}} \sum_{i=1}^{n-1} \sum_{j=i+1}^{n} \sum_{k=1}^{n-1} \sum_{l=k+1}^{n} W_{j}^{n}\left(X_{i}\right) W_{k}^{n}\left(X_{l}\right) \operatorname{cov}\left(e_{i}, e_{k} \mid X_{i}, X_{k}\right) \operatorname{cov}\left(e_{j}, e_{l} \mid X_{j}, X_{l}\right) \\
& +\frac{4}{n^{2}} \sum_{i=1}^{n-1} \sum_{j=i+1}^{n} \sum_{k=1}^{n-1} \sum_{l=k+1}^{n} W_{j}^{n}\left(X_{i}\right) W_{k}^{n}\left(X_{l}\right) \operatorname{cov}\left(e_{i}, e_{l} \mid X_{i}, X_{l}\right) \operatorname{cov}\left(e_{j}, e_{k} \mid X_{j}, X_{k}\right) \\
= & I_{3 e}^{\prime}+I_{3 e}^{\prime \prime}
\end{aligned}
$$


Since both terms are of the same order, it suffices to analyze the first term. As before, this term can be split up in four terms. We will only handle the first term $I_{3 e}^{\prime}$ as the rest are similar.

$$
\begin{aligned}
I_{3 e}^{\prime} & =\frac{4 \sigma^{4}\left(S^{00}\right)^{2}}{n^{4} h^{2}} \sum_{i=1}^{n-1} \sum_{j=i+1}^{n} \sum_{k=1}^{n-1} \sum_{l=k+1}^{n} \frac{K\left(\frac{X_{j}-X_{i}}{h}\right) K\left(\frac{X_{k}-X_{l}}{h}\right)}{f\left(X_{i}\right) f\left(X_{l}\right)} \rho_{n}\left(X_{i}-X_{k}\right) \rho_{n}\left(X_{j}-X_{l}\right) \\
& =\frac{4 \sigma^{4}\left(S^{00}\right)^{2}}{h^{2}} \iiint \int K\left\{\frac{(v-r)+(r-u)}{h}\right\} K\left(\frac{t-r}{h}\right) \rho_{n}(u-t) \rho_{n}(v-r) f(v) f(t) d u d t d v d r\left\{1+o_{p}(1)\right\} \\
& \leq 4 \sigma^{4}\left(S^{00}\right)^{2} f_{\max }^{2} \iiint \int K(\alpha) K(\beta)\left|\rho_{n}(h \alpha+r-u)\right|\left|\rho_{n}(u-r-h \beta)\right| d \alpha d \beta d u d r\left\{1+o_{p}(1)\right\} \\
& \leq 4 \sigma^{4}\left(S^{00}\right)^{2} K_{\max }^{2} f_{\max }^{2} \iint\left\{\int\left|\rho_{n}(h \alpha+r-u)\right| d \alpha\right\}\left\{\int\left|\rho_{n}(u-r-h \beta)\right| d \beta\right\} d u d r\left\{1+o_{p}(1)\right\} .
\end{aligned}
$$

Let $\delta>1$, then the inner integral yields after a change of variable

$$
\int\left|\rho_{n}(h \alpha+r-u)\right| d \alpha=\frac{1}{n^{\delta} h}\left\{n^{\delta} \int\left|\rho_{n}(t)\right| d t\right\} \leq \frac{\rho_{\delta}}{n^{\delta} h} .
$$

We have that

$$
I_{3 e}^{\prime} \leq \frac{4 \sigma^{4}\left(S^{00}\right)^{2} K_{\max }^{2} f_{\max }^{2} \rho_{\delta}^{2} \mu^{2}(\Omega)}{n^{2 \delta} h^{2}}=O\left(\frac{1}{n^{2 \delta} h^{2}}\right) .
$$

Finally, combining all results gives

$$
\operatorname{var}\left(I_{3} \mid X_{1}, \ldots, X_{n}\right)=O_{p}\left(\frac{1}{n^{2 \delta} h^{2}}\right)
$$

The theorem follows immediately from (5), (6), (8) and (9)

$$
\begin{aligned}
\operatorname{RSS}(h)-\operatorname{ASE}(h)= & \frac{1}{n} \sum_{i=1}^{n} e_{i}^{2}+O_{p}\left(\frac{h^{p+1}}{\sqrt{n}}\right)-\frac{2 \sigma^{2} S^{00} K(0) \mu(\Omega)}{n h}\left\{1+o_{p}(1)\right\} \\
& -\frac{2 \sigma^{2} S^{00} K(0) \rho_{c}}{n h}+O_{p}\left(\frac{1}{n^{\delta} h}\right) \\
= & \frac{1}{n} \sum_{i=1}^{n} e_{i}^{2}-\frac{2 \sigma^{2} S^{00} K(0)\left\{\mu(\Omega)+\rho_{c}\right\}}{n h}\left\{1+o_{p}(1)+O_{p}\left(\sqrt{n} h^{p+2}\right)+O_{p}\left(n^{1-\delta}\right)\right\} \\
= & \frac{1}{n} \sum_{i=1}^{n} e_{i}^{2}-\frac{2 \sigma^{2} S^{00} K(0)\left\{\mu(\Omega)+\rho_{c}\right\}}{n h}+o_{p}\left\{n^{-(2 p+2) /(2 p+3)}\right\}
\end{aligned}
$$

for $h \in \mathcal{H}_{n}$ and $n \rightarrow \infty$.

\section{AdDitionAl SIMULATION RESULTS}

These results are complementary to the simulation results of Section 5 of the paper. For the same data generation process as described in Section 5, Figures 1 and 2, Figure 3 and 4 illustrate the results for the autoregressive model of order one and two error structure respectively for a sample size 50 and 200.

Below we compare the performance of the kernel with $K(0)=0$, the proposed method (see Eq. 6 of the paper) and two popular methods. One is the plugin bandwidth selector proposed by Francisco-Fernández et al. (2004) and the other is to ignore the correlation among the errors 

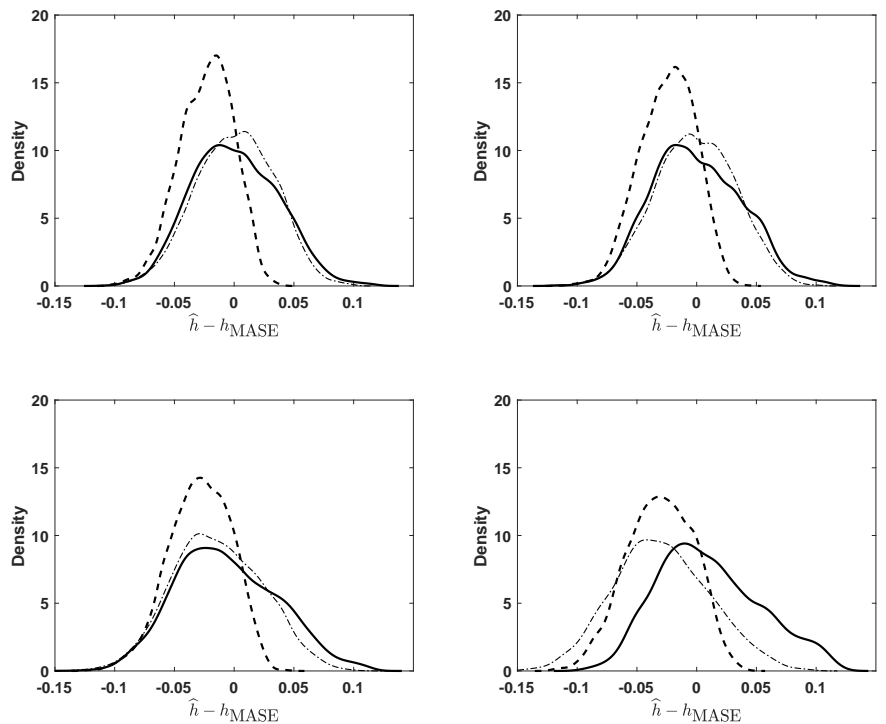

Fig. 1: Densities of the bandwidth estimators $\widehat{h}$ (solid line), plug-in bandwidth $\widehat{h}_{\text {plgn }}$ (dashed line) and cross-validation ignoring correlation $\widehat{h}_{\text {norm }}$ (dash dotted line) for autoregressive model of order one correlated errors and $n=50$ with $\alpha=1000$ (top left), $\alpha=200$ (top right), $\alpha=100$ (bottom left) and $\alpha=50$ (bottom right).

and simply use a Gaussian kernel with standard cross-validation. The same kernels were taken as in Section 5. We conducted 1000 simulations of the data generation process: Consider the model

$$
Y_{i}=m\left(X_{i}\right)+e_{i}
$$

with regression function (Opsomer et al., 2001)

$$
m(X)=1-6 X+36 X^{2}-53 X^{3}+22 X^{5}
$$

with $X \sim U[0,1], \quad e \sim N(0,0.5)$ and $\operatorname{cov}\left(e_{i}, e_{j} \mid X_{i}, X_{j}\right)=\sigma^{2} \rho\left(X_{i}-X_{j}\right)$ with $\rho(t)=$ $\exp (-\alpha|t|)$ and $\alpha=\{50,100,200,1000, \infty\}$. Our comparison criterion is the asymptotic mean squared error for each run of the simulation. These are then averaged over all 1000 runs to obtain the mean asymptotic squared error. Table 1 shows the mean asymptotic squared error results for the four bandwidth selection methods with their respective standard deviation for a sample size $n=200$. When there is little to no correlation, the kernel with $K(0)=0$ results in a higher error rate than the three other methods which is due to the non-optimality of the kernel. Next, consider the quasi-periodic autoregressive model of order 2

$$
\rho(t)=\exp \left(-\alpha_{1} t / 2\right) \cos (p t)+\frac{\alpha_{1}}{2 p} \sin (p t),
$$

where $p^{2}=\left(\alpha_{2}-\alpha_{1}^{2} / 4\right)>0$. The regression function and the data generation process are the same as before. Table 2 shows the mean asymptotic squared error results for the three bandwidth selection methods with their respective standard deviation for a sample size $n=200$. A typical result of the regression estimation for the quasi-periodic autoregressive model of order two is shown in Figure 5. This illustrates the ability of the kernel with $K(0)=0$. As supported by 

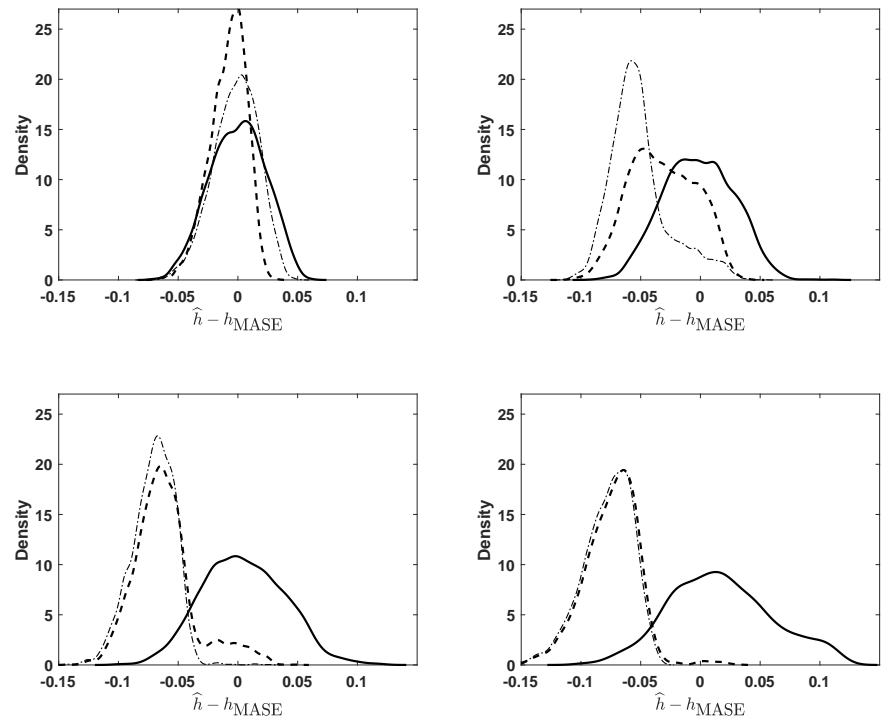

Fig. 2: Densities of the bandwidth estimators $\widehat{h}$ (solid line), plug-in bandwidth $\widehat{h}_{\text {plgn }}$ (dashed line) and cross-validation ignoring correlation $\widehat{h}_{\text {norm }}$ (dash dotted line) for autoregressive model of order one correlated errors and $n=200$ with $\alpha=1000$ (top left), $\alpha=200$ (top right), $\alpha=100$ (bottom left) and $\alpha=50$ (bottom right).

Table 1: Mean asymptotic squared error $\left(\times 10^{-1}\right)$, based on 1000 simulations, for four different bandwidth selection methods for sample size $n=200$. The standard deviation $\left(\times 10^{-1}\right)$ is reported between parenthesis

\begin{tabular}{|c|c|c|c|c|}
\hline & $K(0$ & $d$ & in & Sta \\
\hline & $0 \cdot 30(0 \cdot 14)$ & $0 \cdot 1$ & $0.21(0.08)$ & 0 . \\
\hline & $0 \cdot 38$ & 0.21 & $0 \cdot 2$ & \\
\hline & 0.7 & $0 \cdot 3$ & $0 \cdot 3$ & \\
\hline & $1.05(0.48)$ & $0 \cdot 6$ & 0.6 & 2. \\
\hline & $1.66(0.72)$ & $1 \cdot 10(0.70)$ & $1.10(0.70)$ & $3 \cdot 80(1)$ \\
\hline
\end{tabular}

theory, this kernel removes the effects of correlated errors but suffers from introducing extra mean squared error into the regression estimate.

Table 2: Mean asymptotic squared error $\left(\times 10^{-1}\right)$, based on 1000 simulations, for four different bandwidth selection methods for sample size $n=200$. The standard deviation $\left(\times 10^{-1}\right)$ is reported between parenthesis

$$
\begin{array}{ccccc} 
& K(0)=0 & \text { Proposed } & \text { Plugin } & \text { Standard CV } \\
\alpha_{1}=50, \alpha_{2}=3000 & 0 \cdot 63(0 \cdot 26) & 0 \cdot 27(0 \cdot 13) & 0 \cdot 30(0 \cdot 14) & 4 \cdot 86(1 \cdot 10) \\
\alpha_{1}=50, \alpha_{2}=4000 & 0 \cdot 56(0 \cdot 25) & 0 \cdot 17(0 \cdot 09) & 0 \cdot 20(0 \cdot 10) & 1 \cdot 10(1 \cdot 12) \\
\alpha_{1}=60, \alpha_{2}=3000 & 0 \cdot 32(0 \cdot 13) & 0 \cdot 26(0 \cdot 12) & 0 \cdot 28(0 \cdot 12) & 4 \cdot 89(1 \cdot 08) \\
\alpha_{1}=60, \alpha_{2}=4000 & 0 \cdot 79(0 \cdot 30) & 0 \cdot 37(0 \cdot 20) & 0 \cdot 40(0 \cdot 19) & 4 \cdot 75(1 \cdot 03)
\end{array}
$$



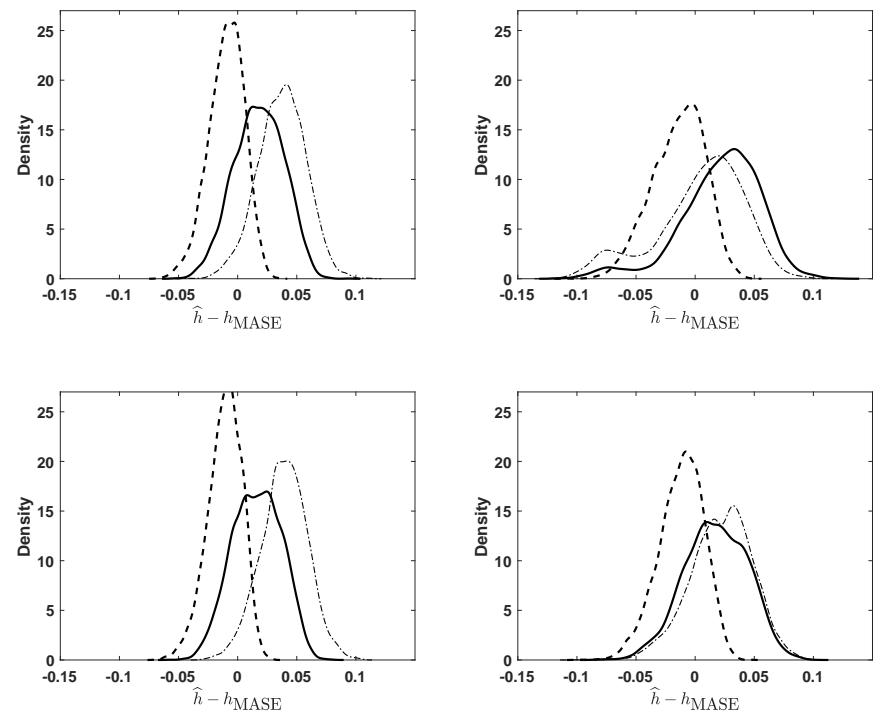

Fig. 3: Densities of the bandwidth estimators $\widehat{h}$ (solid line), plug-in bandwidth $\widehat{h}_{\text {plgn }}$ (dashed line) and cross-validation ignoring correlation $\widehat{h}_{\text {norm }}$ (dash dotted line) for autoregressive model of order two correlated errors and $n=50$ with $\alpha_{1}=50, \alpha_{2}=3000$ (top left), $\alpha_{1}=50, \alpha_{2}=4000$ (top right), $\alpha_{1}=60, \alpha_{2}=3000$ (bottom left) and $\alpha_{1}=60, \alpha_{2}=4000$ (bottom right).

Finally, we illustrate that our methodology still holds in practice even when the data are generated from a density with unbounded support. As before, we conducted 1000 simulations of the data generation process: Consider the model

$$
Y_{i}=m\left(X_{i}\right)+e_{i}, \quad i=1, \ldots, 500
$$

with regression function

$$
m(X)=0.1 e^{-X}+2 e^{-X^{2}}
$$

with $X \sim N(0,1), e \sim N(0,0.5)$ and $\operatorname{cov}\left(e_{i}, e_{j} \mid X_{i}, X_{j}\right)=\sigma^{2} \rho\left(X_{i}-X_{j}\right)$ with $\rho(t)=$ $\exp (-\alpha|t|)$ and $\alpha=50$. Table 3 shows the mean asymptotic squared error results for the four bandwidth selection methods with their respective standard deviation for a sample size $n=200$. A typical result is shown in Figure 6.

Table 3: Mean asymptotic squared error $\left(\times 10^{-1}\right)$, based on 1000 simulations, for four different bandwidth selection methods for sample size $n=500$ and $\alpha=50$. The standard deviation $\left(\times 10^{-1}\right)$ is reported between parenthesis

$$
\begin{array}{lccc}
K(0)=0 & \text { Proposed } & \text { Plugin } & \text { Standard CV } \\
0 \cdot 73(0 \cdot 28) & 0 \cdot 29(0 \cdot 16) & 0 \cdot 35(0 \cdot 18) & 1.45(1 \cdot 20)
\end{array}
$$



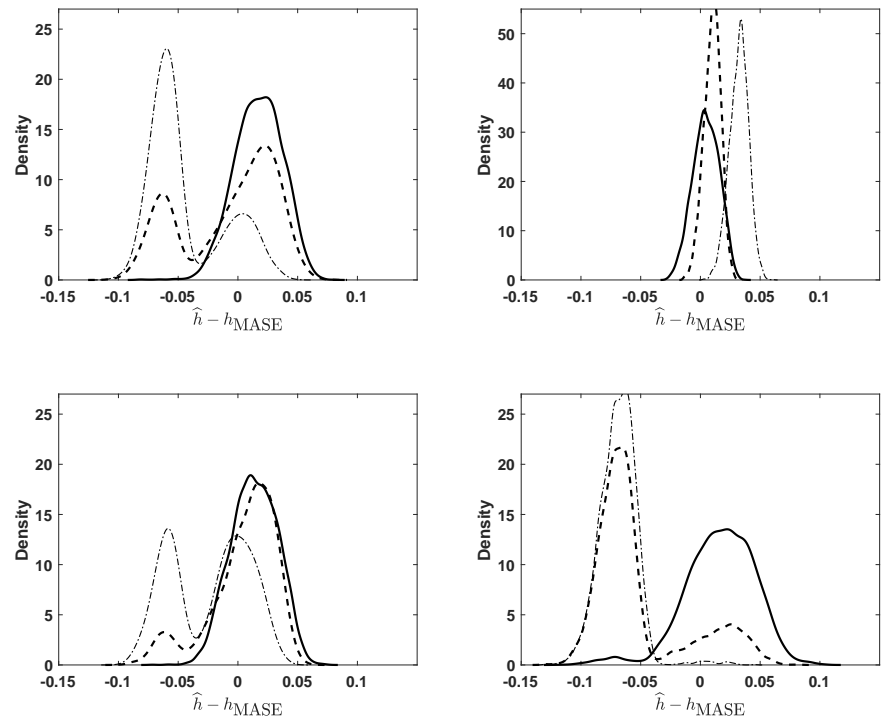

Fig. 4: Densities of the bandwidth estimators $\widehat{h}$ (solid line), plug-in bandwidth $\widehat{h}_{\text {plgn }}$ (dashed line) and cross-validation ignoring correlation $\widehat{h}_{\text {norm }}$ (dash dotted line) for autoregressive model of order two correlated errors and $n=200$ with $\alpha_{1}=50, \alpha_{2}=3000$ (top left), $\alpha_{1}=50, \alpha_{2}=4000$ (top right), $\alpha_{1}=60, \alpha_{2}=3000$ (bottom left) and $\alpha_{1}=60, \alpha_{2}=4000$ (bottom right). Note that the top right figure has a different scale on the vertical axis.

\section{REFERENCES}

FAn, J. \& GiJbels, I. (1996). Local Polynomial Modelling and Its Applications. New York: Chapman \& Hall/CRC. Francisco-Fernández, M., Opsomer, J. D. \& Vilar-Fernández, J. M. (2004). Plug-in bandwidth selector for local polynomial regression estimator with correlated errors. J. Nonparametr. Stat., 16, 127-151.

J. Opsomer, Y. WANG \& Y. YANG (2001). Nonparametric regression with correlated errors. Statist. Sci., 16, $134-153$. 


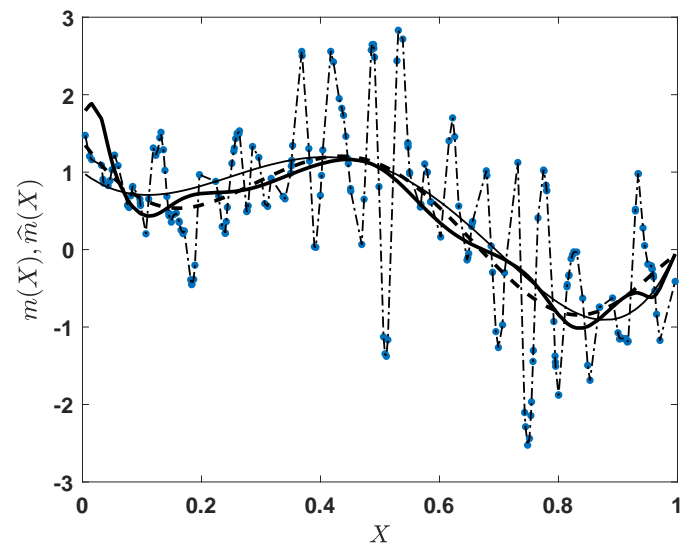

Fig. 5: Regression estimation with quasi-periodic autoregressive model of order $2\left(\alpha_{1}=\right.$ $50, \alpha_{2}=3000$ ). True function (full line), standard cross-validation with Gaussian kernel ignoring correlation (dash dotted line), bandwidth selection method based on a kernel with $K(0)=0$ (bold line), bandwidth selection method based on a kernel with $K(0)=0$ and corrected for a Gaussian kernel via Eq. 6 (bold dashed line).

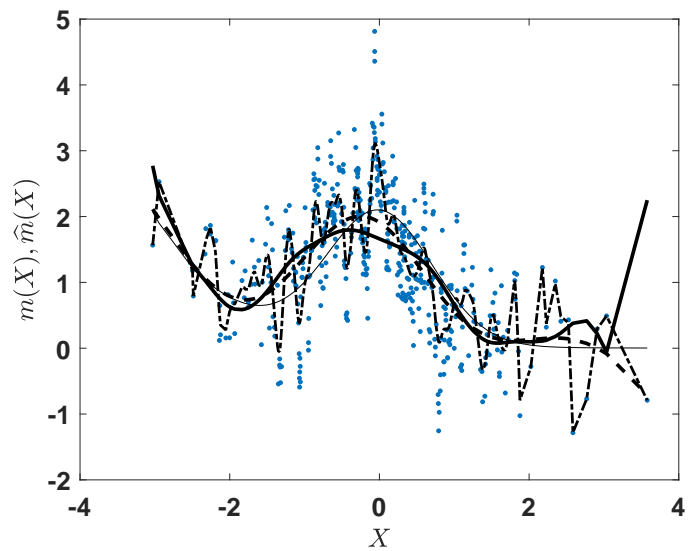

Fig. 6: Regression estimation with autoregressive model of order $1(\alpha=50)$ and $n=500$. True function (full line), standard cross-validation with Gaussian kernel ignoring correlation (dash dotted line), bandwidth selection method based on a kernel with $K(0)=0$ (bold line), bandwidth selection method based on a kernel with $K(0)=0$ and corrected for a Gaussian kernel via Eq. 6 (bold dashed line). 